\title{
Dynamic instabilities in scalar neural field equations with space-dependent delays
}

\author{
N A Venkov and S Coombes and P C Matthews \\ School of Mathematical Sciences, University of Nottingham, Nottingham, NG7 \\ 2RD, UK.
}

\begin{abstract}
In this paper we consider a class of scalar integral equations with a form of spacedependent delay. These non-local models arise naturally when modelling neural tissue with active axons and passive dendrites. Such systems are known to support a dynamic (oscillatory) Turing instability of the homogeneous steady state. In this paper we develop a weakly nonlinear analysis of the travelling and standing waves that form beyond the point of instability. The appropriate amplitude equations are found to be the coupled mean-field Ginzburg-Landau equations describing a Turing-Hopf bifurcation with modulation group velocity of $O(1)$. Importantly we are able to obtain the coefficients of terms in the amplitude equations in terms of integral transforms of the spatio-temporal kernels defining the neural field equation of interest. Indeed our results cover not only models with axonal or dendritic delays but those which are described by a more general distribution of delayed spatiotemporal interactions. We illustrate the predictive power of this form of analysis with comparison against direct numerical simulations, paying particular attention to the competition between standing and travelling waves and the onset of Benjamin-Feir instabilities.
\end{abstract}

Key words: neuronal networks, integral equations, space dependent delays, dynamic pattern formation, travelling waves, amplitude equations. 


\section{Introduction}

The ability of neural field models to exhibit complex spatio-temporal dynamics has been studied intensively since their introduction by Wilson and Cowan [1]. They have found wide application in interpreting experiments in vitro e.g. electrical stimulation of slices of neural tissue [2-5] and phenomena in vivo such as the synchronisation of cortical activity during epileptic seizures [6] or uncovering the mechanism of geometric visual hallucinations [7-9]. The sorts of dynamic behaviour that are typically observed in neural field models includes, spatially and temporally periodic patterns (beyond a Turing instability) $[7,8]$, localised regions of activity (bumps and multi-bumps) [10,11] and travelling waves (fronts, pulses, target waves and spirals) $[12,13]$. The equations describing the evolution of the activity in the neural field typically take the form of integro-differential or integral equations. A variety of modifications have been put forward adding various biological mechanisms to the original model. For a recent review see [14].

It is the purpose of this paper to consider in more detail the role of axonal and dendritic delays in generating novel spatio-temporal patterns. Specifically, we are interested in patterns emerging via Turing-type instabilities of the homogeneous steady state. There are four different types of instability that generically occur, giving rise to i) shift to a new uniform stationary state, ii) stationary periodic patterns, iii) uniform global (bulk) oscillations and iv) travelling (oscillatory) periodic patterns. We shall refer to i) and ii) as static instabilities and iii) and iv) as dynamic. Note that in the original two-population model (without space-dependent delays) developed by Wilson and Cowan [1] there are two time scales and two space-scales: the different membrane time-constants for the excitatory and inhibitory synapses, and the associated spatial interaction scales. However, in order to decrease the dimensionality of the system it is common to assume that inhibitory synapses act much faster than the excitatory. The unequal finite spatial scales are preserved in the form of the connectivity kernel of the new single equation (often of Mexican hat shape), but one of the temporal scales is lost. This is the type of reduced system we consider here, with the notable exception that we bring back another time-scale associated with a space-dependent delay. Space-dependent delays arise naturally through two distinct signal processing mechanisms in models of neural tissue. Axonal delays are associated with the finite speed of action potential propagation. In models with dendrites there is a further distributed delay associated with the arrival of input at a synapse away from the cell-body. It is precisely the inclusion of these biological features that can give 
rise to not only static, but dynamic Turing instabilities. For examples of the treatment of truly two-population models and the possibility of oscillatory pattern formation without space-dependent delays we refer the reader to Tass [8] and Bressloff and Cowan [9].

Axonal delays arise due to the finite speed of action potential propagation in transferring signals between distinct points in the neural field and are modelled in the work of $[15,1,16]$ as simple space-dependent delays. Delays arising from the processing of incoming synaptic inputs by passive dendritic trees may also be incorporated into neural field models, as in the work of Bressloff [17]. In both cases it is now known that these space-dependent delays can lead to a dynamic Turing instability of a homogeneous steady state. These were first found in neural field models by Bressloff [17] for dendritic delays and more recently by Hutt et al. [18] for axonal delays. Both these studies show that a combination of short range inhibition and longer range excitation with a space-dependent delay may lead to a dynamic instability. This choice of connectivity, which we shall call inverted Mexican hat, is natural when considering cortical tissue and remembering that principal pyramidal cells i) are often enveloped by a cloud of inhibitory interneurons, and ii) that long range cortical connections are typically excitatory [19-21]. Detailed examination by linear analysis on the relation between the connectivity shape (the balance between excitation and inhibition) and the dominant pattern type has been done by Hutt [22]. Roxin et al. did similar work for a model with fixed discrete delay [23].

Our main result will be to go beyond the linear analysis of Bressloff [17] and Hutt et al. [18] and to develop amplitude equations for a one dimensional scalar neural field equation with space-dependent delays. Although borrowing heavily from techniques in the PDE community for the weakly nonlinear analysis of states beyond a Turing bifurcation [24-26], our analysis is complicated by the fact that it deals with pattern forming models described by integral equations. Previously amplitude equations in the context of neural field models have been derived also in [27-29]. Importantly we work with integral equations describing neural fields with both axonal and dendritic processing. Although they have existed as models for some time they are far less studied than models lacking such biologically realistic terms. Our formulation is general and encompasses a number of such models. When deriving the amplitude equations we consider also the effects of space-dependant modulation and show that the appropriate equations are the mean-field Ginzburg-Landau equations [30].

In Section 2 we introduce the class of models we shall consider and describe how they can 
be written as integral equations with a particular spatio-temporal convolution structure. Next in Section 3 we analyse the linear stability of the homogeneous steady state and derive the conditions for the onset of a dynamic Turing (Turing-Hopf) instability point. In Section 4 we derive the amplitude equations via a multiple-scales analysis. These amplitude equations are analysed in Section 5 to determine the selection process for travelling as opposed to standing waves. Moreover, we also consider Benjamin-Feir modulational instabilities in which a periodic travelling wave (of moderate amplitude) loses energy to a small perturbation of other waves with nearly the same frequency and wavenumber. $\mathrm{Nu}-$ merical experiments are presented to illustrate and support our analysis of the amplitude equations. In Section 6 we consider the generalisation of our approach to tackle models with a form of spike frequency adaptation. We show that this can strongly influence the selection process for travelling versus standing waves. Finally in Section 7 we discuss other natural extensions of the work we have presented in this paper.

\section{The model}

In many continuum models for the propagation of electrical activity in neural tissue it is assumed that the synaptic input current is a function of the pre-synaptic firing rate function $[31,15,10,32]$. When considering a simple one-dimensional system of identical neurons communicating through excitatory and inhibitory synaptic connections it is typical to arrive at models of the form

$$
u(x, t)=\int_{-\infty}^{\infty} \mathrm{d} y w(x-y) \int_{-\infty}^{t} \mathrm{~d} s \eta(t-s) f(u(y, s-|x-y| / v)) .
$$

Here, $u(x, t)$ is identified as the synaptic activity at position $x \in \mathbb{R}$ at time $t \in \mathbb{R}^{+}$. The firing rate activity generated as a consequence of local synaptic activity is simply $f(u)$, where the firing rate function $f$ is prescribed purely in terms of the properties of some underlying single neuron model or chosen to fit experimental data. A common choice for the firing rate function is the sigmoid

$$
f(u)=(1+\exp (-\beta(u-h)))^{-1}
$$

with steepness parameter $\beta>0$ and threshold $h>0$. The spatial kernel $w(x)=w(|x|)$ describes not only the anatomical connectivity of the network, but also includes the sign of synaptic interaction. For simplicity it is also often assumed to be isotropic and homogeneous, as is the case here. The temporal convolution involving the kernel $\eta(t)(\eta(t)=0$ 

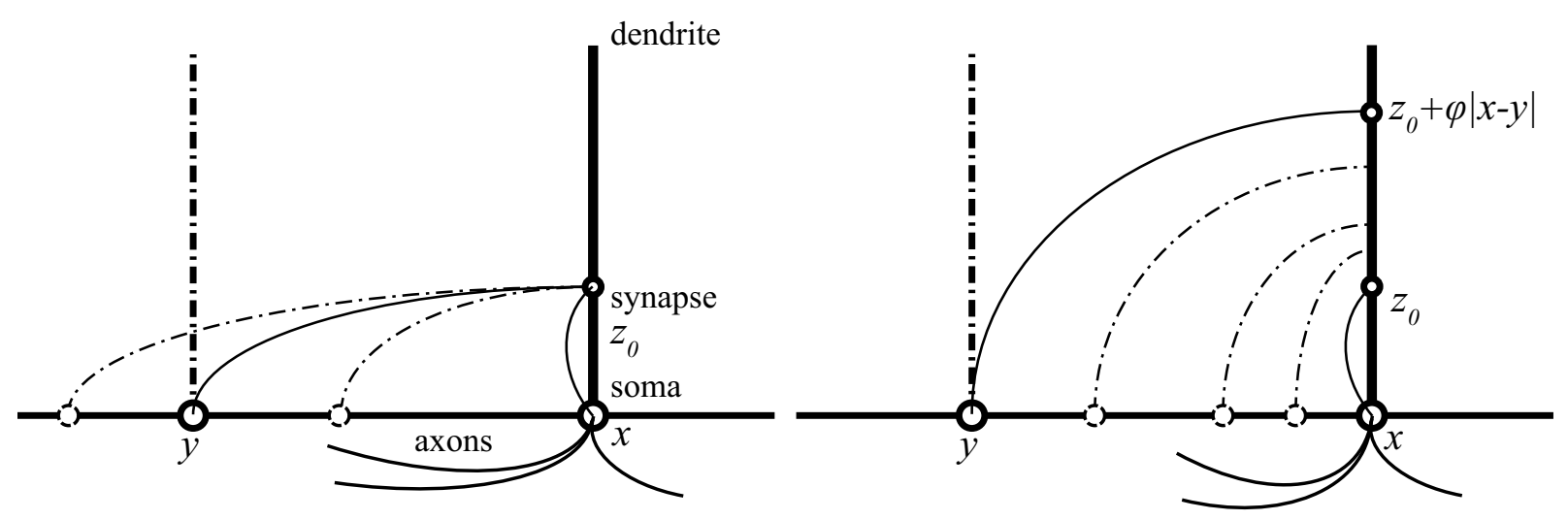

Fig. 1. Examples of two neural field models with dendritic cable that we consider in this paper. Left: All synaptic inputs impinge on the same site at a distance $z_{0}$ from the soma. Right: Here the distance of the synapse from the soma is linearly correlated with the spatial separation $|x-y|$ between neurons.

for $t<0$ ) represents synaptic processing of signals within the network, whilst the delayed temporal argument to $u$ under the spatial integral represents the delay arising from the finite speed of signals travelling between points $x$ and $y$; namely $|x-y| / v$ where $v$ is the velocity of action potential. In models with dendrites there is a further space-dependent delay associated with the processing of inputs at a synapses away from the cell-body. A simple way to incorporate this in a model is to represent the dendritic tree as a single passive cable with diffusive properties (see below).

By introducing the kernel $K(x, t)=w(x) \delta(t-|x| / v)$ we can re-write (1) in the succinct form

$$
u=\eta * K \otimes f \circ u
$$

where we have introduced the two-dimensional convolution operation $\otimes$ :

$$
(K \otimes g)(x, t)=\int_{-\infty}^{\infty} \mathrm{d} s \int_{-\infty}^{\infty} \mathrm{d} y K(x-y, t-s) g(y, s)
$$

for $g=g(x, t)$, and the temporal convolution operation $*$ :

$$
(\eta * h)(t)=\int_{0}^{\infty} \mathrm{d} s \eta(s) h(t-s)
$$

for $h=h(t)$. The form of (3) allows us to naturally generalize a model with axonal delays to one with dendritic delays or any other type of time-dependent connectivity. For example the model of Bressloff $[17,33]$ incorporating a neuron with a semi-infinite dendritic cable 
with potential $v=v(z, t), z \in \mathbb{R}^{+}$is written

$$
\begin{aligned}
& \partial_{t} v=-v / \tau_{D}+D \partial_{z z} v+I(x, z, t), \\
& I(x, z, t)=\int_{-\infty}^{\infty} \mathrm{d} y w(x-y, z) \int_{-\infty}^{\infty} \mathrm{d} s \eta(t-s) f(u(y, s)) .
\end{aligned}
$$

Here $w(x, z)$ is an axo-dendritic connectivity function depending not only upon cell-cell distances $|x|$, but on dendritic distances $z$ also. Assuming that there is no flow of current back from the cell body (soma) at $z=0$ to the dendrite then the neural field equation is simply given by $u(x, t)=v(x, 0, t)$. Hence, this dendritic model is recovered by (3) with the choice $K(x, t)=\int_{0}^{\infty} \mathrm{d} z w(x, z) E(z, t)$, where $E(z, t)=\mathrm{e}^{-t / \tau_{D}} \mathrm{e}^{-z^{2} / 4 D t} / \sqrt{\pi D t}$ is the Green's function of the semi-infinite cable equation (and $E(z, t)=0$ for $t<0$ ). With a single synapse at a fixed distance $z_{0}>0$ from the cell body $w(x, z)=w(x) \delta\left(z-z_{0}\right)$, the generalized connectivity function is separable, taking the form $K(x, t)=w(x) E\left(z_{0}, t\right)$. We will also look at non-separable models with space-dependent dendritic delays $w(x, z)=$ $w(x) \delta\left(z-z_{0}-\phi|x|\right)$. In these models axons from more distant neurons arborize further up the dendritic cable in accordance with anatomical data [34]. See Fig. 1 for an illustration. In fact throughout this paper we shall consider the model as written in the form of (3), and allow for arbitrary choices of $K=K(x, t)$, subject to $K(x, t)=K(|x|, t)$ and $K(x, t)=0$ for $t<0$, such that the classic axonal and dendritic delay models are recovered as special cases. Later in Section 6 we shall consider further generalizations of (3) to include the effects of neuronal modulation and adaptation.

\section{Turing instability analysis}

In this paper we are primarily interested in the analysis of pattern formation beyond a Turing instability for the neural field equation given by (3). It is the dependence of $K$ on the pair $(x, t)$ rather than just $x$, as would be the case in the absence of space-dependent delays, that can give rise to not only static, but dynamic Turing instabilities. Here we briefly re-visit the standard linear Turing instability analysis, along similar lines to that of Bressloff [17] and Hutt et al. [18]. For a general review of pattern formation and pattern forming instabilities we refer the reader to [35-38]. For a discussion of pattern formation within the context of neural field models we suggest the articles by Ermentrout [39] and Bressloff [40].

Let $u(x, t)=\bar{u}$ be the spatially-homogeneous steady state of equation (3). The conditions 
for the growth of inhomogeneous solutions can be obtained through simple linear stability analysis of $\bar{u}$. Namely, we look for instabilities to spatial perturbations of globally periodic type $\mathrm{e}^{i k x}$ and determine the intrinsic wavelength $2 \pi / k$ of the dominant growing mode. From (3) the homogeneous steady state satisfies

$$
\bar{u}=\widetilde{\eta}(0) \widehat{K}(0,0) f(\bar{u})
$$

where we have introduced the following Laplace and Fourier-Laplace integral transforms:

$$
\widetilde{\eta}(\lambda)=\int_{0}^{\infty} \mathrm{d} s \eta(s) \mathrm{e}^{-\lambda s}, \quad \widehat{K}(k, \lambda)=\int_{-\infty}^{\infty} \mathrm{d} y \int_{0}^{\infty} \mathrm{d} s K(y, s) \mathrm{e}^{-(i k y+\lambda s)} .
$$

We Taylor-expand the firing rate function, which is the only nonlinearity in the system, around the steady state $f(u)=f(\bar{u})+\gamma_{1}(u-\bar{u})+\gamma_{2}(u-\bar{u})^{2}+\ldots$ to obtain the linearised model

$$
u-\bar{u}=\gamma_{1} \eta * K \otimes(u-\bar{u}) .
$$

The control parameter for our bifurcation analysis is therefore $\gamma_{1}=f^{\prime}(\bar{u})$. To obtain the conditions for linear stability we consider solutions of the form $u-\bar{u}=\operatorname{Re} \mathrm{e}^{\lambda t+i k x}$ where $\lambda=\nu+i \omega \in \mathbb{C}$. Although the system and its solutions are real, for conciseness we will often work with the complex extension and restrict our attention to the real case when this has implications for the analysis. After substitution into (9) we obtain a dispersion relation for $(k, \lambda)$ in the form $L(k, \lambda)=0$, where

$$
L(k, \lambda)=1-\gamma_{1} \widetilde{\eta}(\lambda) \widehat{K}(k, \lambda)
$$

For a fixed $\gamma_{1}$ solving $L(k, \lambda)=0$ defines a function $\lambda(k)$. An instability occurs when for the first time there are values of $k$ at which the real part of $\lambda$ is nonnegative (see Fig. 2, left). A Turing bifurcation point is defined as the smallest value $\gamma_{c}$ of the control parameter for which there exists some $k_{c} \neq 0$ satisfying $\operatorname{Re}\left(\lambda\left(k_{c}\right)\right)=0$. It is said to be static if $\operatorname{Im}\left(\lambda\left(k_{c}\right)\right)=0$ and dynamic if $\operatorname{Im}\left(\lambda\left(k_{c}\right)\right) \equiv \omega_{c} \neq 0$. The dynamic instability is often referred to as a Turing-Hopf bifurcation and generates a global pattern with wavenumber $k_{c}$, which moves coherently with a speed $c=\omega_{c} / k_{c}$, i.e. as a periodic travelling wave train. If the maximum of the dispersion curve is at $k=0$ then the mode that is first excited is another spatially uniform state. If $\omega_{c} \neq 0$, we expect the emergence of a homogeneous limit cycle with temporal frequency $\omega_{c}$.

For simplicity we will assume that at $\gamma_{1}=\gamma_{c}$ the associated value of $k_{c}$ is unique (up to a sign change). Since $K$ is an even function of the space variable, wave-numbers will always 

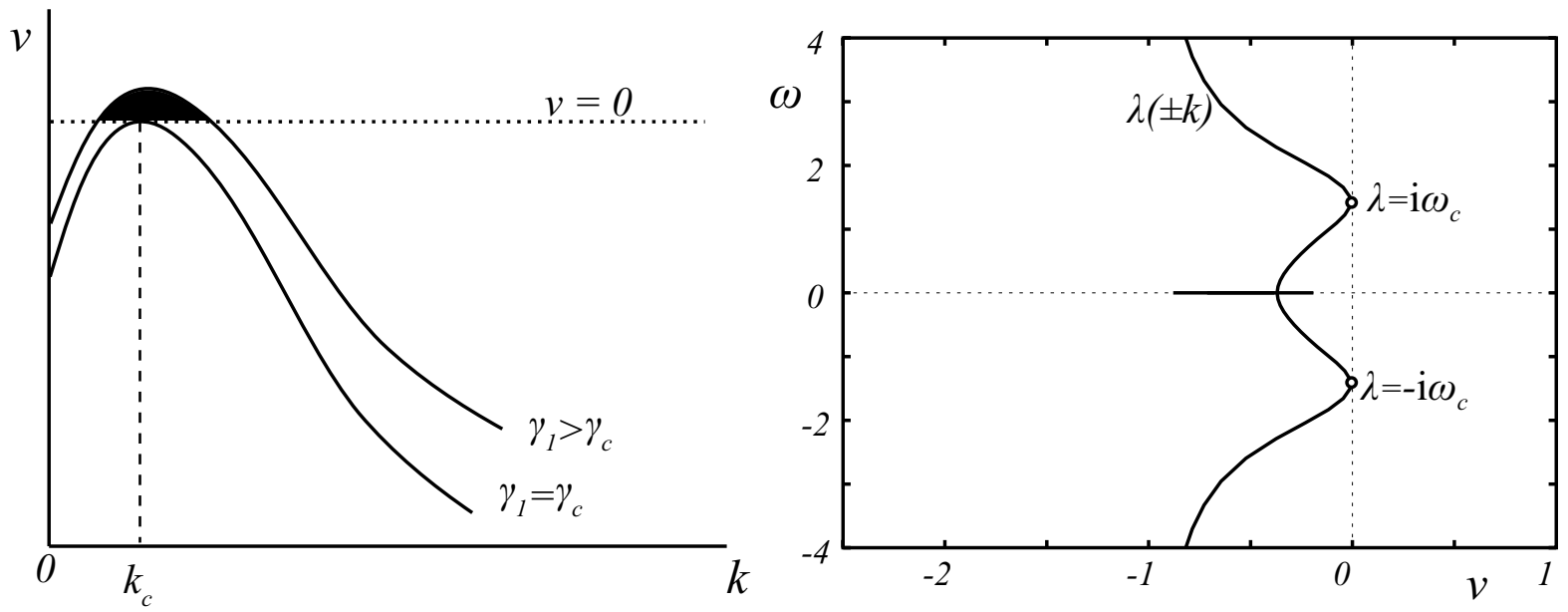

Fig. 2. Left: An illustration of the mechanism for a Turing instability: as $\gamma_{c}$ is increased a range of wavenumbers around $k_{c}$ become linearly unstable. Right: A typical dispersion curve in the complex $(\nu, \omega)$-plane by a complex conjugate pair of roots as $k$ is varied, plotted at the critical value $\gamma_{1}=\gamma_{c}$.

come in pairs $\pm k_{c}$ and because the problem is real the dispersion curve has reflective symmetry around the real axis and frequencies $\pm \omega_{c}$ will also appear together as solutions. Hence, the eigenspace of the linear equation will be real and at $\gamma_{c}$ a complete basis is given by

$$
\left\{\cos \left(\omega_{c} t+k_{c} x\right), \cos \left(\omega_{c} t-k_{c} x\right), \sin \left(\omega_{c} t+k_{c} x\right), \sin \left(\omega_{c} t-k_{c} x\right)\right\} .
$$

Beyond a Turing instability $\left(k_{c} \neq 0\right)$ we expect the growth of patterns of the form $\mathrm{e}^{i\left(k_{c} x \pm \omega_{c} t\right)}+\mathrm{cc}$ (where cc stands for complex conjugate), with $\omega_{c}=0$ for a static instability, $\omega_{c} \neq 0$ for a dynamic instability and $k_{c}=0$ at a Hopf bifurcation. Note that for a dynamic instability, generically giving rise to periodic travelling waves, it may also be possible to see a standing wave, arising from the interaction of a left and right travelling wave. To determine the conditions under which this may occur it is necessary to go beyond a linear analysis and determine the evolution of mode amplitudes. The techniques to do this will be described in Section 4, where we also see that the growing solutions predicted by the linear analysis converge to patterns with certain finite amplitude due to the nonlinear (saturating) properties of the model.

Next we consider some illustrative examples and compare the linear instability predictions with direct numerical simulations. To do this we write $\lambda=\nu+i \omega$ and split the dispersion relation into real and imaginary parts to obtain

$$
G(\nu, \omega)=0, \quad H(\nu, \omega)=0,
$$




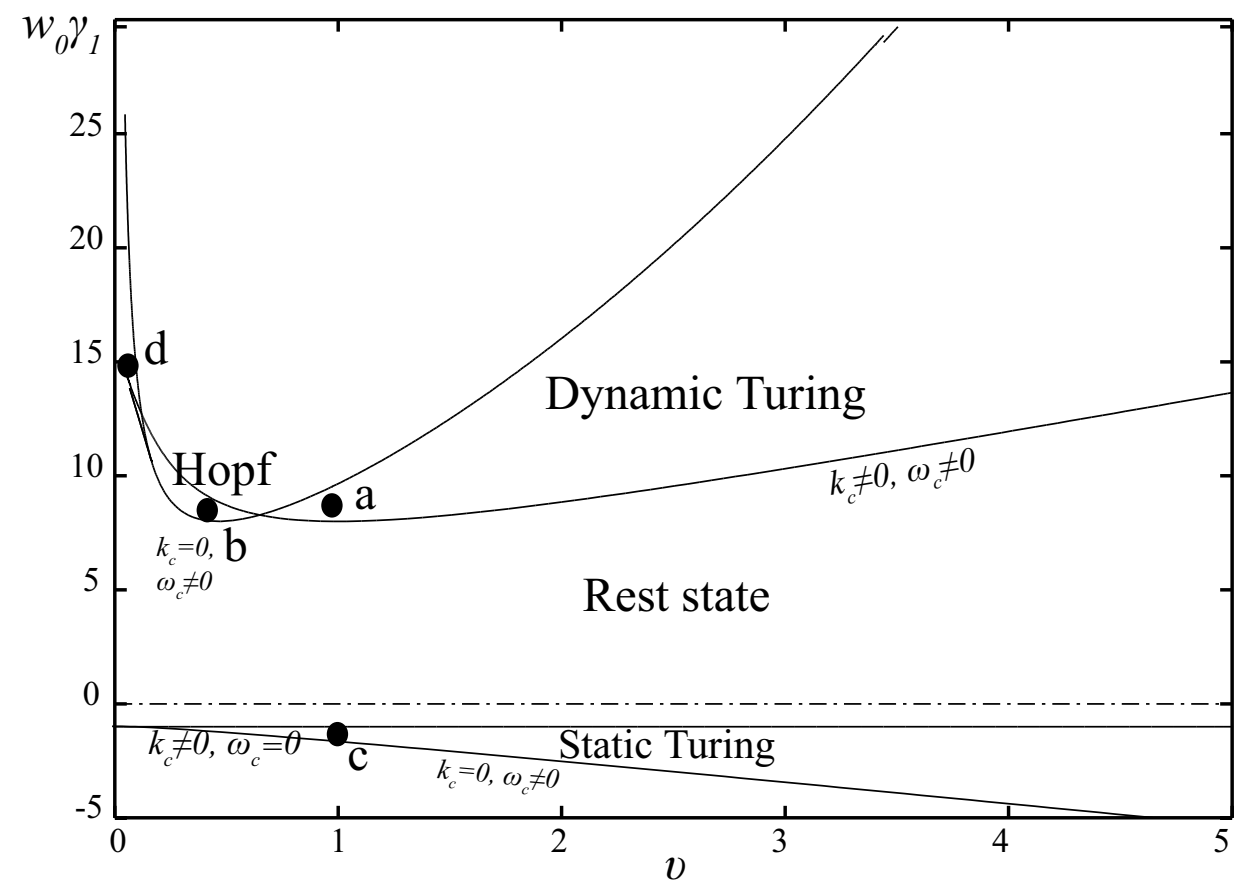

Fig. 3. Critical curves showing the instability threshold $\gamma_{c}$ as a function of the axonal speed $v$ for the model in Section 3.1.

where $G(\nu, \omega)=\operatorname{Re} L(k, \nu+i \omega)$ and $H(\nu, \omega)=\operatorname{Im} L(k, \nu+i \omega)$. Solving the system of equations (12) gives us a curve in the plane $(\nu, \omega)$ parameterised by $k$. A static bifurcation may thus be identified with the tangential intersection of $\omega=\omega(\nu)$ along the line $\nu=0$ at the point $\omega=0$. Similarly a dynamic bifurcation is identified with a tangential intersection at $\omega \neq 0$ (see Fig. 2, right). We are required to track points in parameter space for which $\nu^{\prime}(\omega)=0$. This is equivalent to tracking points where

$$
\partial_{k} G \partial_{\omega} H-\partial_{k} H \partial_{\omega} G=0 .
$$



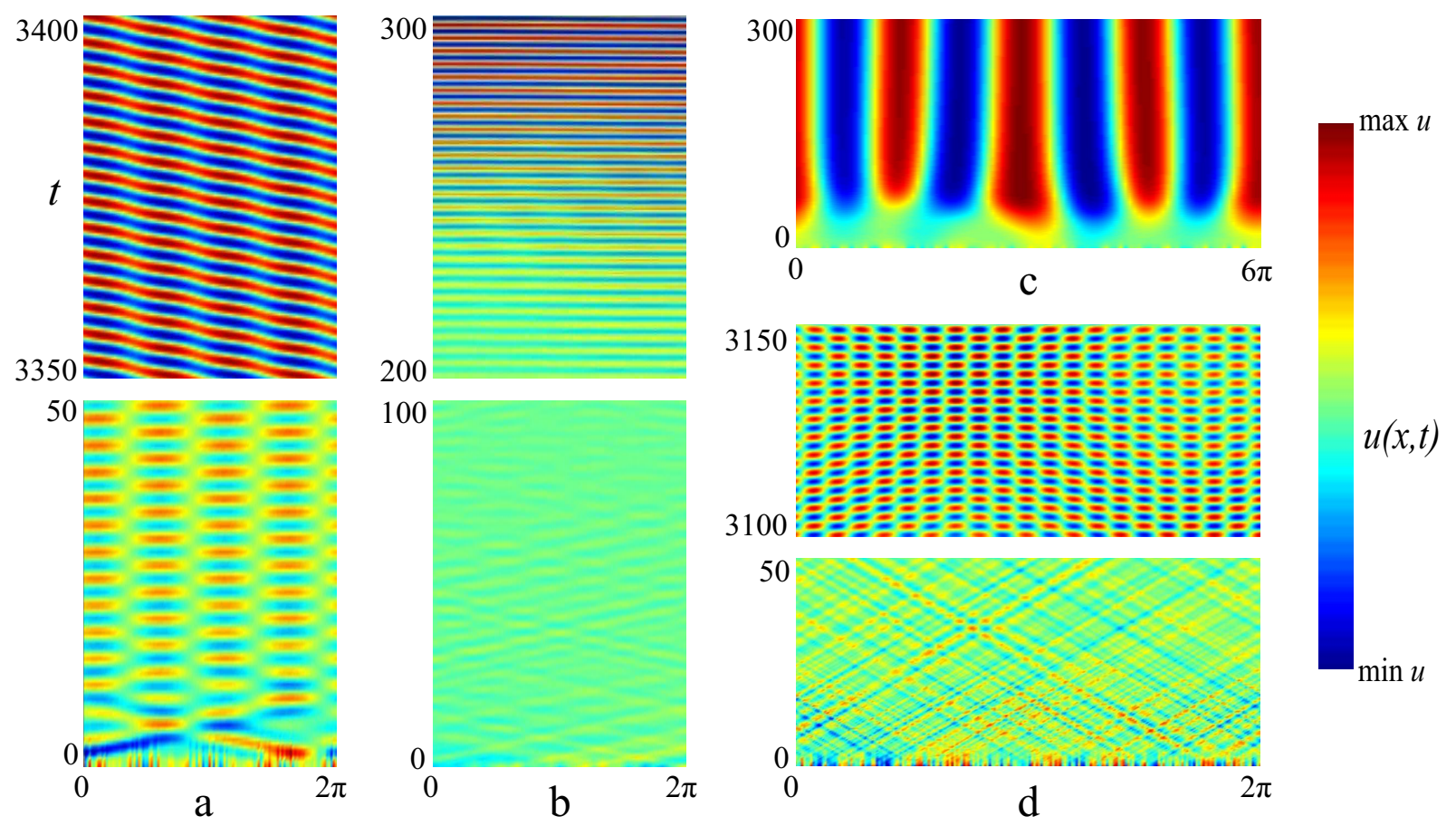

Fig. 4. Space-time plots of solutions corresponding to points a-d in the parameter plot on Fig. 3 with the choice $f(u)=\gamma_{1} u-0.2 u^{3}$. The model is simulated with periodic boundary conditions and random initial conditions. Legend: a. travelling wave (standing wave is initially selected due to the numerical discretization but it is unstable as we will show in sec.5); b. bulk oscillation; c. static pattern; d. large wavenumber waves. Similar results are obtained for other saturating choices of $f$, such as the popular sigmoidal function (2).

\subsection{An example: axonal delays}

We take a wizard hat connectivity function

$$
w(x)=-w_{0}(1-|x|) \mathrm{e}^{-|x|}
$$

with space-dependent axonal delays such that $K(x, t)=w(x) \delta(x-|t| / v)$, as discussed in Section 2. The global weight $w_{0}$ allows us to study both inverted $\left(w_{0}>0\right)$ and standard $\left(w_{0}<0\right)$ wizard hat connectivities in a common framework. The Fourier-Laplace transform of $K(x, t)$ is

$$
\widehat{K}(k, \lambda)=-2 w_{0} \frac{(a-1) a^{2}+k^{2}(a+1)}{\left(a^{2}+k^{2}\right)^{2}}, \quad a=1+\frac{\lambda}{v} .
$$

Further, we choose the alpha function synaptic response $\eta(t)=\alpha^{2} t \mathrm{e}^{-\alpha t} \Theta(t)$ where $\Theta(t)$ is the Heaviside step function, $\Theta(t)=1$ for $t \geq 0$ and $\Theta(t)=0$ otherwise. A simple 
calculation gives $\widetilde{\eta}(\lambda)=(1+\lambda / \alpha)^{-2}$. The dispersion relation (10) can then be written as a 6th-order polynomial of the form $\sum_{n=0}^{6} a_{n}\left(\alpha, v, \gamma_{1}, k\right) \lambda^{n}=0$. The expressions for the coefficients $a_{n}$ are easily calculated, though for brevity we do not list them here. Full details will be given elsewhere [41]. Using numerical continuation we can track the set of solution points $\left(\gamma_{c}, k_{c}, \omega_{c}\right)$ of the system of equations defined by (12) and (13) in system parameter space. Moreover, we can construct a critical curve in $\left(v, \gamma_{1}\right)$-parameter space delimiting the region of stability of the homogeneous steady state. Here we track all six roots of the dispersion relation and find that for $w_{0}>0$ two pairs of complex roots can cross into the positive half-plane with increasing $\gamma_{1}$. One of these pairs crosses with nonzero wavenumber $k_{c}(v)$ and the other with $k_{c}=0$. A plot of all critical curves $\gamma_{1}=\gamma_{c}(v)$ is shown on the left of Fig. 3. An illustration of corresponding solutions is shown Fig. 4. For the standard wizard hat connectivity $\left(w_{0}<0\right)$ we see, as expected, that the static Turing instability is independent of the axonal velocity $v$.

Several important qualitative features for the case $w_{0}>0$ become clear from Fig. 3 . For any finite $v$ there exists a positive threshold $\gamma_{1}=\gamma_{c}(v)$ at which modes with some wavenumber $k_{c}(v)$ become excited; on the other hand, as $v \rightarrow \infty, \gamma_{c}$ also tends to infinity. The latter observation is consistent with the failure of models without axonal delay to demonstrate dynamic pattern formation when the connectivity is of inverted wizard hat type. A dynamic instability is first met for $v=1$ and dominates for medium and large speeds, while for small speeds there is a window of Hopf instability giving rise to spatially coherent periodic oscillations (across the entire domain). As $v$ becomes smaller than 0.1 the pattern wavenumber quickly, though continuously, shifts from $k=0$ to very large values. Direct numerical simulations of the full model shows excellent agreement with the predictions of the linear instability analysis. Some examples are shown in Fig. 3. The techniques used for direct numerical simulations of the models studied in this paper are described in [42].

\subsection{An example: dendritic delays}

We make the same choices of $w(x)$ and $\eta(t)$ for the dendritic model given by (6) as in the example just studied above with axonal delays with a synapse at a fixed distance $z_{0}$ from the soma. In this case the (separable) kernel describing the model is given by $K(x, t)=w(x) E\left(z_{0}, t\right)$. Then the integral transform of $K$ is $\widehat{K}(\lambda, k)=\widehat{w}(k) \widetilde{E}\left(\lambda, z_{0}\right)$. We

have $\widetilde{E}(\lambda, z)=\exp (-z \sqrt{\kappa+\lambda / D}) / D \sqrt{\kappa+\lambda / D}$, where $\kappa=1 /\left(D \tau_{D}\right)$ and a real Fourier 
transform $\widehat{w}(k)=-4 w_{0} k^{2} /\left(1+k^{2}\right)^{2}$ independent of $\lambda$. Hence, the critical wavenumber at all instabilities is constant $k_{c}=1$. Although there are countably many eigenvalues only two pairs can ever cross into the positive plane. The Turing instability curve for $w_{0}$ as a function of $z_{0}$ is shown in Fig. 5, left. If we consider the synaptic contact point to be linearly correlated with the distance between neuronal sites, then $K(x, t)=w(x) E\left(z_{0}+\phi|x|, t\right)$. In this case $\widehat{K}(x, \lambda)$ is given by (15) with $a=1+\phi \sqrt{\kappa+\lambda}$. Note that to further include axonal delays we would simply write $a=1+\lambda / v+\phi \sqrt{\kappa+\lambda}$. Comparing the results from Fig. 3 (left) and Fig. 5 (right) we see that, as expected, qualitatively there are strong similarities between a model with purely axonal delays and one without axonal delays possessing dendritic delays correlated to the distance between neuronal sites.

\section{Weakly nonlinear analysis}

A characteristic feature of the dynamics of systems beyond an instability is the slow growth of the dominant eigenmode, giving rise to the notion of a separation of scales. This observation is key in deriving the so-called amplitude equations. In this approach information about the short-term behaviour of the system is discarded in favour of a description on some appropriately identified slow time scale. By Taylor-expansion of the dispersion curve near its maximum one expects the scalings $\nu \sim \gamma_{1}-\gamma_{c}, k-k_{c} \sim \sqrt{\gamma_{1}-\gamma_{c}}$,
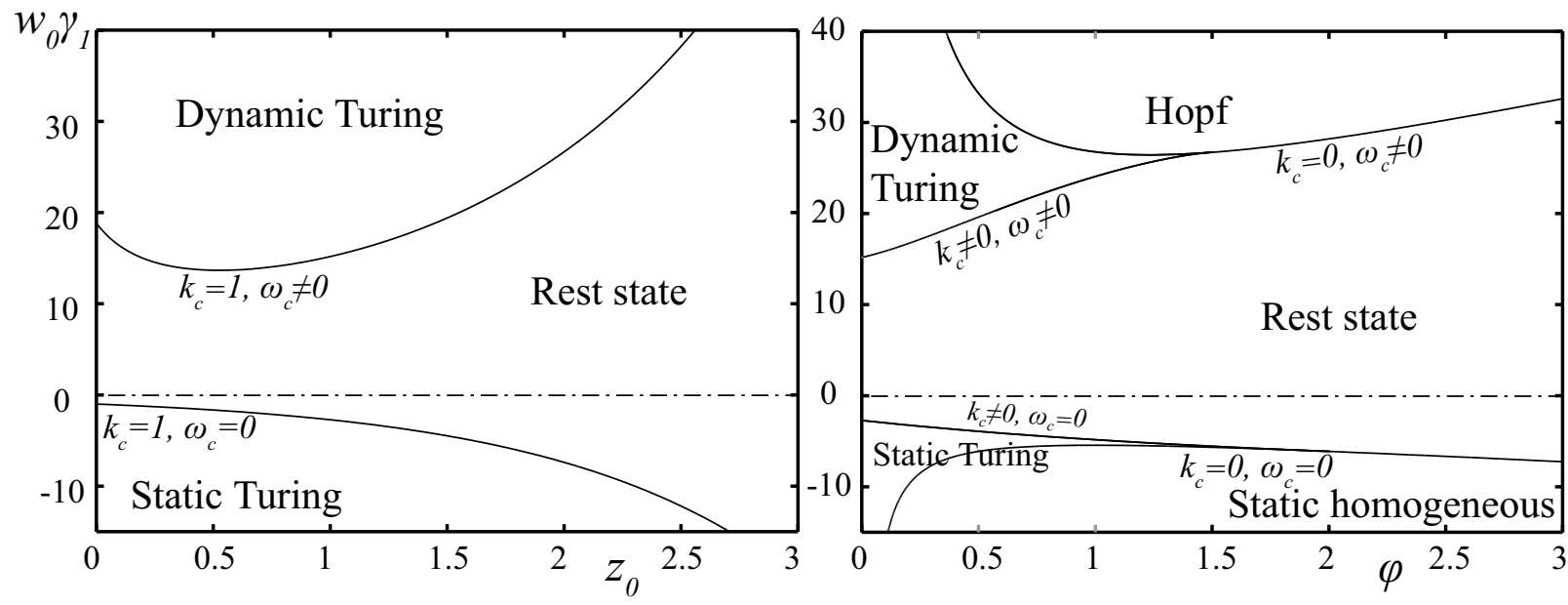

Fig. 5. The Turing instability curves for a model with a passive dendritic cable. Left: With fixed synaptic contact point at a distance $z_{0}$ from the cell body. Right: With synaptic contact point correlated with the distance between neuronal sites by $z_{0}+\phi|x|, z_{0}=1$. Other parameters are $\alpha=\tau=D=1$. 
close to bifurcation, where $\gamma_{1}$ is the bifurcation parameter. Since the eigenvectors at the point of instability are of the type $A_{1} \mathrm{e}^{i\left(\omega_{c} t+k_{c} x\right)}+A_{2} \mathrm{e}^{i\left(\omega_{c} t-k_{c} x\right)}+c c$, for $\gamma_{1}>\gamma_{c}$ emergent patterns are described by an infinite sum of unstable modes (in a continuous band) of the form $\mathrm{e}^{\nu_{0}\left(\gamma_{1}-\gamma_{c}\right) t} \mathrm{e}^{i\left(\omega_{c} t+k_{c} x\right)} \mathrm{e}^{i k_{0} \sqrt{\gamma_{1}-\gamma_{c}} x}$. Let us denote $\gamma_{1}=\gamma_{c}+\epsilon^{2} \delta$ where $\epsilon$ is arbitrary and $\delta$ is a measure of the distance from the bifurcation point. Then, for small $\epsilon$ we can separate the dynamics into fast eigen-oscillations $\mathrm{e}^{i\left(\omega_{c} t+k_{c} x\right)}$, and slow modulations of the form $\mathrm{e}^{\nu_{0} \epsilon^{2} t} \mathrm{e}^{i k_{0} \epsilon x}$. If we set as further independent variables $\tau=\epsilon^{2} t$ for the modulation timescale and $\chi=\epsilon x$ for the long-wavelength spatial scale (at which the interactions between excited nearby modes become important) we may write the weakly nonlinear solution as $A_{1}(\chi, \tau) \mathrm{e}^{i\left(\omega_{c} t+k_{c} x\right)}+A_{2}(\chi, \tau) \mathrm{e}^{i\left(\omega_{c} t-k_{c} x\right)}+\mathrm{cc}$. It is known from the standard theory [35] that weakly nonlinear solutions will exist in the form of either travelling waves $\left(A_{1}=0\right.$ or $\left.A_{2}=0\right)$ or standing waves $\left(A_{1}=A_{2}\right)$.

We are now in a position to derive the envelope or amplitude equations for patterns emerging beyond the point of Turing-Hopf instability for the neural field model (3). Here we omit the simpler case for the static Turing instability since it has already been analysed in [43]. For a PDE system the general form of the amplitude equations can be found very easily by considering the resonances in the system. Indeed the normal form of the Turing-Hopf bifurcation is well-known to be a system of two coupled complex Ginzburg-Landau equations [37]. To tackle an integral system we will use the lengthy but systematic approach of multiple scales analysis. By doing so we ultimately obtain the normal form for a bifurcation to patterns that have a non-zero group velocity. This type of normal form was first found in [30] in the context of travelling wave convection.

\subsection{Scale separation}

Introducing the further intermediate time scale $\theta=\epsilon t$, solutions to (3) satisfy

$$
u\left(x, t, \epsilon x, \epsilon t, \epsilon^{2} t\right)=\int_{-\infty}^{t} \mathrm{~d} r \eta(t-r) \int_{-\infty}^{\infty} \mathrm{d} y \int_{-\infty}^{\infty} \mathrm{d} s K(x-y, r-s) f \circ u\left(y, s, \epsilon y, \epsilon s, \epsilon^{2} s\right) .
$$

Note that the operator on the right-side of (16) is not identical to the convolution $\eta * K \otimes$. Here the integration acts on the last three variables as well, due to their $\epsilon$-dependence on $x$ and $t$. In order to find the equation describing the slow dynamics we have to assess this $\epsilon$-dependent contribution. Here we will adopt an approach that considers a polynomial expansion of the right hand side of (16) in powers of $\epsilon$. The coefficients of this polynomial are convolutions each acting at a single scale only. We begin by writing the solution as 
an asymptotic expansion

$$
u-\bar{u}=\epsilon u_{1}+\epsilon^{2} u_{2}+\epsilon^{3} u_{3}+\ldots
$$

with, as yet, unknown functions $u_{i}=u_{i}(x, t)$. Further, we substitute the firing rate function $f$ by its Taylor-expansion $f(u)=f(\bar{u})+\gamma_{1}(u-\bar{u})+\gamma_{2}(u-\bar{u})^{2}+\ldots$ where the coefficients $\gamma_{2}, \gamma_{3}, \ldots$ remain as free parameters that will participate in the final amplitude equations. When we consider specific models in Section 5 we will map their firing rate parametrizations and restrict these coefficients. To separate the scales at which the integration acts, we write $\epsilon y=\epsilon y+\epsilon x-\epsilon x=\chi+\epsilon(y-x)$, and similarly for $\epsilon s$ and $\epsilon^{2} s$ :

$$
u_{i}\left(y, s, \epsilon y, \epsilon s, \epsilon^{2} s\right)=u_{i}\left(y, s, \chi+\epsilon(y-x), \theta+\epsilon(s-t), \tau+\epsilon^{2}(s-t)\right) .
$$

This enables us to make a Taylor expansion in the last three arguments to get

$$
\begin{array}{r}
u_{i}\left(y, s, \epsilon y, \epsilon s, \epsilon^{2} s\right)=u_{i}(y, s, \chi, \theta, \tau)+\epsilon\left[(y-x) \frac{\partial}{\partial \chi}+(s-t) \frac{\partial}{\partial \theta}\right] u_{i}(y, s, \chi, \theta, \tau)+ \\
\epsilon^{2}\left[\frac{1}{2}\left((y-x) \frac{\partial}{\partial \chi}+(s-t) \frac{\partial}{\partial \theta}\right)^{2}+(s-t) \frac{\partial}{\partial \tau}\right] u_{i}(y, s, \chi, \theta, \tau)+O\left(\epsilon^{3}\right) .
\end{array}
$$

The slow variables $\chi, \theta, \tau$ are independent of $y$ or $s$ on which the integration acts and we have a sum of convolutions of the type $\eta * K \otimes$. In order to write down these convolutions it is convenient to introduce the notation:

$$
\begin{gathered}
\eta^{t}(t)=-t \eta(t), \quad \eta^{t t}(t)=t^{2} \eta(t), \quad K^{x}(x, t)=-x K(x, t), \quad K^{t}(x, t)=-t K(x, t), \\
K^{x t}(x, t)=x t K(x, t), \quad K^{x x}(x, t)=x^{2} K(x, t), \quad K^{t t}(x, t)=t^{2} K(x, t) .
\end{gathered}
$$


Then by using $s-t=(r-t)+(s-r)$ (to step through the intermediate time scale), we can write for each $u_{i}$

$$
\begin{aligned}
& \int_{-\infty}^{t} \mathrm{~d} r \eta(t-r) \int_{-\infty}^{\infty} \mathrm{d} y \int_{-\infty}^{\infty} \mathrm{d} s \eta(t-r) K(x-y, r-s) u_{i}\left(y, s, \epsilon y, \epsilon s, \epsilon^{2} s\right)= \\
& \left\{\eta * K \otimes u_{i}+\epsilon\left(\eta * K^{x} \otimes \frac{\partial}{\partial \chi}+\left(\eta^{t} * K+\eta * K^{t}\right) \otimes \frac{\partial}{\partial \theta}\right) u_{i}\right. \\
& +\frac{\epsilon^{2}}{2}\left[\eta * K^{x x} \otimes \frac{\partial^{2}}{\partial \chi^{2}}+2\left(\eta^{t} * K^{x}+\eta * K^{x t}\right) \otimes \frac{\partial^{2}}{\partial \chi \partial \theta}\right. \\
& \left.\left.+\left(\eta^{t t} * K+2 \eta^{t} * K^{t}+\eta * K^{t t}\right) \otimes \frac{\partial^{2}}{\partial \theta^{2}}+2\left(\eta^{t} * K+\eta * K^{t}\right) \otimes \frac{\partial}{\partial \tau}\right] u_{i}\right\}(x, t, \chi, \theta, \tau) \\
& +O\left(\epsilon^{3}\right) \\
& \equiv M_{0} u_{i}+\epsilon M_{1} u_{i}+\epsilon^{2} M_{2} u_{i}+O\left(\epsilon^{3}\right) .
\end{aligned}
$$

We denote by $M_{j}$ the segregated action of operators (identified above) on the different scales. Hence, the model (3) separates into an infinite sequence of equations (which we truncate at third order) of the form:

$$
\begin{aligned}
& u_{1}=M_{0}\left(\gamma_{c} u_{1}\right) \\
& u_{2}=M_{0}\left(\gamma_{c} u_{2}+\gamma_{2} u_{1}^{2}\right)+M_{1}\left(\gamma_{c} u_{1}\right), \\
& u_{3}=M_{0}\left(\gamma_{c} u_{3}+2 \gamma_{2} u_{1} u_{2}+\gamma_{3} u_{1}^{3}+\delta u_{1}\right)+M_{1}\left(\gamma_{c} u_{2}+\gamma_{2} u_{1}^{2}\right)+M_{2}\left(\gamma_{c} u_{1}\right) .
\end{aligned}
$$

\subsection{Fredholm alternative}

One can see that each equation above contains terms of the asymptotic expansion of $u$ only of the same order or lower. This means that we can start from the first equation and systematically solve for $u_{i}$. In fact, if we set $\mathcal{L}=I-\gamma_{c} M_{0}=I-\gamma_{c} \eta * K \otimes$ the system $(22),(23),(24)$ has the general form $\mathcal{L} u_{n}=g_{n}\left(u_{1}, u_{2}, \ldots, u_{n-1}\right)$ and the right-hand side $g_{n}$ will always contain known quantities. Thus to construct the solutions of any finite truncation of the full system describing all the $u_{i}$ it is enough to know the inverse of the linear operator $\mathcal{L}$.

In terms of $\mathcal{L}$ the first equation $(22)$ is $\mathcal{L} u_{1}=0$ and we see that our entire solution space is a perturbation of the kernel of $\mathcal{L}$. In fact, we have already solved (22) in Section 3 and know that ker $\mathcal{L}=\operatorname{span}\left\{\cos \left(\omega_{c} t+k_{c} x\right), \cos \left(\omega_{c} t-k_{c} x\right), \sin \left(\omega_{c} t+k_{c} x\right), \sin \left(\omega_{c} t-k_{c} x\right)\right\}$, i.e. 
any solution $u_{1} \in \operatorname{ker} \mathcal{L}$ can be expressed as a linear combination

$$
u_{1}=A_{1}(\chi, \theta, \tau) \mathrm{e}^{i\left(\omega_{c} t+k_{c} x\right)}+A_{2}(\chi, \theta, \tau) \mathrm{e}^{i\left(\omega_{c} t-k_{c} x\right)}+c c,
$$

where $A_{1,2}$ are arbitrary complex coefficients depending only on the slow space and time scales. We may now use the Fredholm alternative [44] to consider restrictions on the $g_{n}$ that will give us equations for the amplitudes $A_{1,2}$.

Since $\mathcal{L}$ acts on the $O(1)$ variables $x$ and $t$ where the solutions $u_{i}$ are periodic we can restrict our attention to the subspace of bounded functions of period $2 \pi / k_{c}$ in $x$ and $2 \pi / \omega_{c}$ in $t$. Let $\Lambda=\left[0,2 \pi / k_{c}\right] \times\left[0,2 \pi / \omega_{c}\right]$ be the periodicity domain and define the inner product of two periodic functions $u(x, t)$ and $v(x, t)$ to be

$$
<u, v>=\frac{k_{c} \omega_{c}}{4 \pi^{2}} \int_{\Lambda} \bar{u}(x, t) v(x, t) \mathrm{d} x \mathrm{~d} t
$$

where the bar denotes complex conjugation. Introducing the functions $\eta^{*}(t)=\eta(-t)$ and $K^{*}(x, t)=K(x,-t)(\eta$ and $K$ reflected around the point $t=0)$, it can be shown (using Fourier series) that the adjoint of $\mathcal{L}$ is the operator $\mathcal{L}^{*}=I-\gamma_{c} \eta^{*} * K^{*} \otimes$, with respect to the inner product (26).

The operator $\mathcal{L}^{*}$ has the same kernel space as $\mathcal{L}$ (since the dispersion relation is invariant under the change $t \rightarrow-t$ ). From the Fredholm alternative we have for all $u \in \operatorname{ker} \mathcal{L}$ that $<u, g_{n}>=<u, \mathcal{L} u_{n}>=<\mathcal{L}^{*} u, u_{n}>=0$. Therefore we can derive equations for the amplitudes simply by calculating the two complex projections

$$
<\mathrm{e}^{i\left(\omega_{c} t \pm k_{c} x\right)}, g_{n}>=0
$$

To simplify notation we set $u^{ \pm}=\mathrm{e}^{i\left(\omega_{c} t \pm k_{c} x\right)}$. Then, the scalar products (27) expand as

$$
<u^{ \pm}, g_{2}>=\gamma_{2} \widetilde{\eta} \widehat{K}<u^{ \pm}, u_{1}^{2}>+\gamma_{c} \mathcal{M}_{1}^{ \pm}\left(\widetilde{\eta} \widehat{K}<u^{ \pm}, u_{1}>\right)=0
$$

and

$$
\begin{gathered}
<u^{ \pm}, g_{3}>=2 \gamma_{2} \widetilde{\eta} \widehat{K}<u^{ \pm}, u_{1} u_{2}>+\gamma_{3} \widetilde{\eta} \widehat{K}<u^{ \pm}, u_{1}^{3}>+\delta \widetilde{\eta} \widehat{K}<u^{ \pm}, u_{1}>+ \\
\mathcal{M}_{1}^{ \pm}\left(\gamma_{c} \widetilde{\eta} \widehat{K}<u^{ \pm}, u_{2}>+\gamma_{2} \widetilde{\eta} \widehat{K}<u^{ \pm}, u_{1}^{2}>\right)+\gamma_{c} \mathcal{M}_{2}^{ \pm}\left(\widetilde{\eta} \widehat{K}<u^{ \pm}, u_{1}>\right)=0 .
\end{gathered}
$$

Here the Fourier-Laplace transforms (see $(8)) \widetilde{\eta}(\lambda)$ and $\widehat{K}(k, \lambda)$ are all evaluated at the points $k=k_{c}$ and $\lambda=i \omega_{c}$. By $\mathcal{M}_{j}^{ \pm}$we denote the differential operators

$$
\mathcal{M}_{1}^{ \pm}=\mp \frac{\partial}{\partial i k} \frac{\partial}{\partial \chi}+\frac{\partial}{\partial i \omega} \frac{\partial}{\partial \theta}, \quad \mathcal{M}_{2}^{ \pm}=\frac{1}{2}\left(\mathcal{M}_{1}^{ \pm}\right)^{2}+\frac{\partial}{\partial i \omega} \frac{\partial}{\partial \tau} \text {. }
$$


Since from $(25) u_{1}$ is itself a linear combination of the modes $u^{ \pm}$, most scalar products in (28) and (29) are easily found: $<u^{+}, u_{1}>=A_{1},<u^{-}, u_{1}>=A_{2},<u^{ \pm}, u_{1}^{2}>=0$, and

$$
<u^{+}, u_{1}^{3}>=3 A_{1}\left(\left|A_{1}\right|^{2}+2\left|A_{2}\right|^{2}\right), \quad<u^{-}, u_{1}^{3}>=3 A_{2}\left(2\left|A_{1}\right|^{2}+\left|A_{2}\right|^{2}\right) .
$$

The first solvability condition $(28)$ reduces to $\mathcal{M}_{1}^{ \pm}\left(\widetilde{\eta} \widehat{K} A_{1,2}\right)=0$, namely

$$
\frac{\partial(\widetilde{\eta} \widehat{K})}{\partial i k} \frac{\partial A_{1,2}}{\partial \chi}= \pm \frac{\partial(\widetilde{\eta} \widehat{K})}{\partial i \omega} \frac{\partial A_{1,2}}{\partial \theta}
$$

The general solutions of these first-order PDEs are

$$
A_{1}(\chi, \theta, \tau)=A_{1}\left(\chi+v_{g} \theta, \tau\right)=A_{1}\left(\xi_{+}, \tau\right), \quad A_{2}(\chi, \theta, \tau)=A_{2}\left(\chi-v_{g} \theta, \tau\right)=A_{2}\left(\xi_{-}, \tau\right)
$$

where $\xi_{ \pm}=\chi \pm v_{g} \theta$ and $v_{g}=\partial \omega / \partial k$. This means that all slow amplitude modulations $A_{1,2}(\xi, \tau)$ will be travelling with an intermediate group speed $v_{g}$ to the left and right respectively. Notice that the repeated action of $\mathcal{M}_{1}^{ \pm}$does not result in zero. We have

$$
\frac{1}{2} \mathcal{M}_{2}^{ \pm}\left(\widetilde{\eta} \widehat{K} A_{1,2}\right)=\frac{1}{2}\left(\mp \frac{\partial}{\partial i k} \pm v_{g} \frac{\partial}{\partial i \omega}\right)^{2}(\widetilde{\eta} \widehat{K}) \cdot \frac{\partial^{2} A_{1,2}}{\partial \xi_{ \pm}^{2}}=d \frac{\partial^{2} A_{1,2}}{\partial \xi_{ \pm}^{2}} \neq 0
$$

To calculate the last remaining product $<u^{ \pm}, u_{1} u_{2}>$ in (29) we need to find $u_{2}$ first. We can do this from the 2nd order equation (23). Since all operators in this equation are linear, $u_{2}$ will be some quadratic form of the basis vectors which we write as $\sum_{m, n \in\{0, \pm 1, \pm 2\}} B_{m, n} \mathrm{e}^{i\left(m \omega_{c} t+n k_{c} x\right)}$. To find the unknown $B_{m, n}$ we substitute this into (23) and match coefficients to obtain

$$
B_{00}=\frac{\gamma_{2} 2\left(\left|A_{1}\right|^{2}+\left|A_{2}\right|^{2}\right) \widetilde{\eta}(0) \widehat{K}(0,0)}{1-\gamma_{c} \widetilde{\eta}(0) \widehat{K}(0,0)},
$$

and

$$
B_{m n}=\frac{\gamma_{2} 2^{\frac{\| m|-| n||}{2}} A_{1}^{\frac{m+n}{2}} A_{2}^{\frac{m-n}{2}} \widetilde{\eta}\left(i m \omega_{c}\right) \widehat{K}\left(n k_{c}, i m \omega_{c}\right)}{1-\gamma_{c} \widetilde{\eta}\left(i m \omega_{c}\right) \widehat{K}\left(n k_{c}, i m \omega_{c}\right)}
$$

for $\{m, n=0, \pm 2\} \backslash(m, n)=(0,0)$. All other coefficients are zero, except for $\left\{B_{m n} \mid m, n=\right.$ $\pm 1\}$ which cannot be determined using this approach because their corresponding modes are in ker $\mathcal{L}$. The undetermined coefficients $B_{1 \pm 1}\left(\xi_{+}, \xi_{-}, \tau\right)$ are acted on by $\mathcal{M}_{1}$ in $(29)$ in the following fashion

$$
\mathcal{M}_{1}^{ \pm}\left(\widetilde{\eta} \widehat{K} B_{1 \pm 1}\right)=\mp 2 \frac{\partial(\widetilde{\eta} \widehat{K})}{\partial i k} \frac{\partial B_{1 \pm 1}}{\partial \xi_{\mp}}
$$


and this expression will appear in the amplitude equation. By virtue of (35) and (36) the last remaining scalar products are calculated as

$$
\begin{aligned}
& <u^{+}, u_{1} u_{2}>=2 \gamma_{2} A_{1}\left(\left|A_{1}\right|^{2}\left(C_{00}+\frac{1}{2} C_{22}\right)+\left|A_{2}\right|^{2}\left(C_{00}+C_{02}+C_{20}\right)\right), \\
& <u^{-}, u_{1} u_{2}>=2 \gamma_{2} A_{2}\left(\left|A_{2}\right|^{2}\left(C_{00}+\frac{1}{2} C_{2-2}\right)+\left|A_{1}\right|^{2}\left(C_{00}+C_{0-2}+C_{20}\right)\right),
\end{aligned}
$$

with

$$
C_{m n}=\frac{\widetilde{\eta}\left(i m \omega_{c}\right) \widehat{K}\left(n k_{c}, i m \omega_{c}\right)}{1-\gamma_{c} \widetilde{\eta}\left(i m \omega_{c}\right) \widehat{K}\left(n k_{c}, i m \omega_{c}\right)} .
$$

We are now in position to write out the third order solvability condition which gives the amplitude equations.

\subsection{The amplitude equations}

Substituting in (29) all necessary scalar products (calculated above) we obtain

$$
\begin{aligned}
& A_{1}\left(\delta \gamma_{c}^{-1}+b\left|A_{1}\right|^{2}+c\left|A_{2}\right|^{2}\right)-2 \frac{\partial(\widetilde{\eta} \widehat{K})}{\partial i k} \frac{\partial B_{11}}{\partial \xi_{-}}+\gamma_{c} d \frac{\partial^{2} A_{1}}{\partial \xi_{+}^{2}}+\gamma_{c} \frac{\partial(\widetilde{\eta} \widehat{K})}{\partial i \omega} \frac{\partial A_{1}}{\partial \tau}=0 \\
& A_{2}\left(\delta \gamma_{c}^{-1}+b\left|A_{2}\right|^{2}+c\left|A_{1}\right|^{2}\right)+2 \frac{\partial(\widetilde{\eta} \widehat{K})}{\partial i k} \frac{\partial B_{1-1}}{\partial \xi_{+}}+\gamma_{c} d \frac{\partial^{2} A_{2}}{\partial \xi_{-}^{2}}+\gamma_{c} \frac{\partial(\widetilde{\eta} \widehat{K})}{\partial i \omega} \frac{\partial A_{2}}{\partial \tau}=0
\end{aligned}
$$

with

$$
b=\gamma_{c}^{-1}\left(3 \gamma_{3}+2 \gamma_{2}^{2}\left(2 C_{00}+C_{22}\right)\right), \quad c=2 \gamma_{c}^{-1}\left(3 \gamma_{3}+2 \gamma_{2}^{2}\left(C_{00}+C_{02}+C_{20}\right)\right) .
$$

To eliminate the unknown coefficients $B_{1 \pm 1}$ we follow [30] and average the two equations in the $\xi_{-}$and $\xi_{+}$variables, respectively. For example, the only quantities in the first equation that vary with $\xi_{-}$are $B_{11}$ and $A_{2}$. However the average $\left\langle B_{11}\right\rangle$ will be independent of $\xi_{-}$ and thus the derivative term vanishes. After averaging we obtain

$$
\begin{aligned}
& \frac{\partial A_{1}}{\partial \tau}=A_{1}\left(a+b\left|A_{1}\right|^{2}+c\left\langle\left|A_{2}\right|^{2}\right\rangle\right)+d \frac{\partial^{2} A_{1}}{\partial \xi_{+}^{2}} \\
& \frac{\partial A_{2}}{\partial \tau}=A_{2}\left(a+b\left|A_{2}\right|^{2}+c\left\langle\left|A_{1}\right|^{2}\right\rangle\right)+d \frac{\partial^{2} A_{2}}{\partial \xi_{-}^{2}}
\end{aligned}
$$


with appropriately modified parameters:

$$
\begin{aligned}
a & =-\delta D \\
b & =-D\left(3 \gamma_{3}+2 \gamma_{2}^{2}\left(2 C_{00}+C_{22}\right)\right) \\
c & =-2 D\left(3 \gamma_{3}+2 \gamma_{2}^{2}\left(C_{00}+C_{02}+C_{20}\right)\right) \\
d & =-\frac{\gamma_{c}^{2}}{2} D\left(\frac{\partial}{\partial i k}-v_{g} \frac{\partial}{\partial i \omega}\right)^{2}(\widetilde{\eta} \widehat{K}) \\
D & =\gamma_{c}^{-2}\left(\frac{\partial(\widetilde{\eta} \widehat{K})}{\partial i \omega}\right)^{-1}
\end{aligned}
$$

The system of equations (44) are the coupled mean-field Ginzburg-Landau equations describing Turing-Hopf bifurcation with modulation group velocity $v_{g}$ of order one $[30,45,46]$. As a reminder of the interpretation of terms in the above equations note that $\widetilde{\eta}=$ $\widetilde{\eta}\left(i \omega_{c}\right), \widehat{K}=\widehat{K}\left(k_{c}, i \omega_{c}\right)$ are the integral transforms (8) of the kernels specifying the model, $\delta=\left(\gamma_{1}-\gamma_{c}\right) / \epsilon^{2}$ is a measure of the distance from the bifurcation point, and $\gamma_{1}, 2 \gamma_{2}, 6 \gamma_{3}$ are the first, second and third derivatives of the nonlinear firing rate function $f(u)$ taken at the homogeneous solution $\bar{u}$. The only parameters that do not appear in the linearised model are $\gamma_{2}, \gamma_{3}$ and they remain as additional degrees of freedom once we have fixed a bifurcating solution to study. The dependent variables $A_{1}\left(\xi_{-}, \tau\right)$ and $A_{2}\left(\xi_{+}, \tau\right)$ are respectively the right and left-going amplitude modulation wavetrains, moving with group velocities $\pm v_{g}$. The definition of the averaging $\langle A\rangle$ depends on the type of solutions we are looking for - localised or periodic. Here we will assume $A_{1,2}$ are periodic with periods $P_{1,2}$. In this case

$$
\left\langle\left|A_{1,2}\right|^{2}\right\rangle=\frac{1}{P_{1,2}} \int_{0}^{P_{1,2}}\left|A_{1,2}\right|^{2} \mathrm{~d} \xi_{\mp} .
$$

The form of the spatial cross-interaction term in (44) reflects the fact that since $v_{g}=O(1)$, as the two wavetrains move across each other they are not able to feel each other's spatial structure.

In the next section we show how to use the normal form to predict the formation of travelling or standing waves beyond the point of Turing instability.

\section{$5 \quad$ Normal form analysis}

The coupled complex Ginzburg-Landau equations are one of the most-studied systems in applied mathematics, with links to nonlinear waves, second-order phase transitions, 
Rayleigh-Bénard convection and superconductivity. They give a normal form for a large class of bifurcations and nonlinear wave phenomena in spatially extended systems and exhibit a large variety of solutions. Many of these appear due to the influence of the boundary conditions. Here our focus is on infinitely-extended systems. First let us neglect the diffusive terms in (44), which allows us to determine the conditions for the selection of travelling vs standing wave behaviour beyond a dynamic Turing instability.

\subsection{Travelling wave versus standing wave selection}

The space-independent reduction of (44) takes the form

$$
\begin{aligned}
& A_{1}^{\prime}=A_{1}\left(a+b\left|A_{1}\right|^{2}+c\left|A_{2}\right|^{2}\right), \\
& A_{2}^{\prime}=A_{2}\left(a+b\left|A_{2}\right|^{2}+c\left|A_{1}\right|^{2}\right) .
\end{aligned}
$$

Such a reduction is valid in the limit of fast diffusion, and is particularly relevant to the interpretation of numerical experiments on a periodic domain (with fundamental period an integer multiple of $\left.2 \pi / k_{c}\right)$. Stationary states of this reduced system can be identified with the homogeneous steady state, travelling wave and standing wave solution of the neural field model (3). The travelling wave solution corresponding to $A_{2}=0$, has a speed $\left(\omega_{c}+\epsilon^{2} e_{1}\right) / k_{c}$ and takes the form

$$
u(x, t)=2 \sqrt{-\frac{a_{r}}{b_{r}}} \cos \left(\left(\omega_{c}+\epsilon^{2} e_{1}\right) t-k_{c} x\right),
$$

with small frequency change $e_{1}=a_{i}-a_{r} b_{i} / b_{r}$. The subscripts $r$ and $i$ denote real and imaginary parts respectively. The standing wave, $A_{1}=A_{2}$ is described by

$$
u(x, t)=4 \sqrt{\frac{-a_{r}}{b_{r}+c_{r}}} \cos k_{c} x \cos \left(\left(\omega_{c}+\epsilon^{2} e_{2}\right) t\right),
$$

with $e_{2}=a_{i}-a_{r}\left(b_{i}+c_{i}\right) /\left(b_{r}+c_{r}\right)$. The linear stability analysis of the stationary states leads to the following conditions for selection between travelling wave (TW) or standing wave $(\mathrm{SW})$ :

$$
\begin{aligned}
a_{r}>0, \quad b_{r}<0, & \text { TW exists (supercritical) and for } b_{r}-c_{r}>0 \text { it is stable, } \\
a_{r}>0, \quad b_{r}+c_{r}<0, & \text { SW exists (supercritical) and for } b_{r}-c_{r}<0 \text { it is stable. }
\end{aligned}
$$

In the parameter regions where these stationary states of the ODE system (47) do not exist, the amplitude of the bifurcating solution remains finite if the nonlinearity $f$ is 

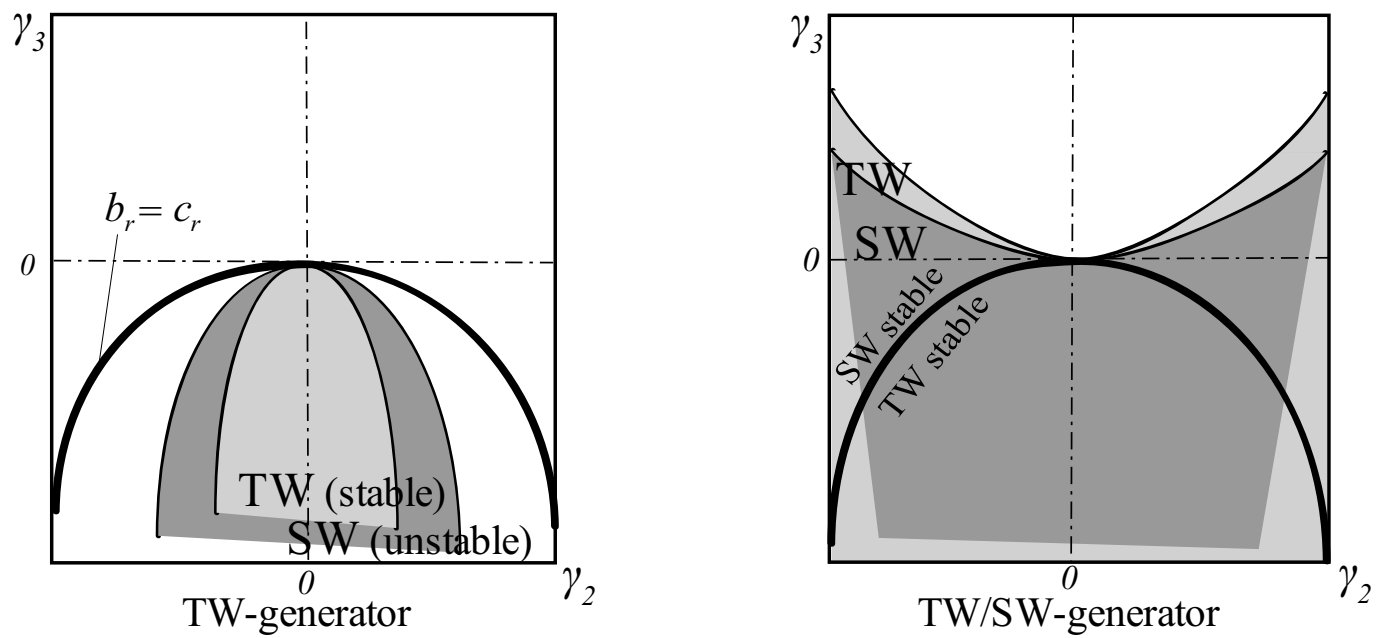

Fig. 6. The two possible configurations of the parameter space $\left(\gamma_{2}, \gamma_{3}\right)$ for standing vs travelling wave selection. $\gamma_{2}$ and $\gamma_{3}$ are the second and third coefficients respectively of the Taylor expansion of the nonlinearity around the homogeneous steady state. Left: This configuration is what we term a TW-generator and occurs for $f^{>}>\dot{g}$. Right: The configuration corresponding to a TW/SW-generator $\left(f^{\prime}<g\right.$ ). Lighter grey denotes the existence of TWs and the darker of SWs. The thick line denotes where $b_{r}=c_{r}$. Where both TWs and SWs exist it forms a selection border in the sense that on one side of it TWs are stable, and on the other SWs are stable.

a bounded function. However, the bifurcation is subcritical and higher-order terms are needed in the normal form to give us information about the amplitudes. We will not concern ourselves with these regions. We now translate the conditions above into the language of our neural field model. Since the only free variables are those describing the nonlinearity $f(u)$, i.e. $\gamma_{2}$ and $\gamma_{3}$, this motivates us to write out $a_{r}, b_{r}, c_{r}$, using (45), as

$$
\begin{aligned}
& a_{r}=\delta \dot{e}, \\
& b_{r}=3 \gamma_{3} \dot{e}+2 \gamma_{2}^{2} f, \quad \text { with } \\
& \dot{e}=\operatorname{Re} D, \quad f^{\prime}=\operatorname{Re}\left(D\left(2 C_{00}+C_{22}\right)\right), \\
& c_{r}=6 \gamma_{3} \dot{e}+4 \gamma_{2}^{2} \dot{g} \text {, } \\
& \dot{g}=\operatorname{Re}\left(D\left(C_{00}+C_{02}+C_{20}\right)\right) \text {. }
\end{aligned}
$$

The expressions $e^{\prime}, f, g$ involve only the kernel transforms and the first derivative of the nonlinearity $f$ which is fixed by the condition defining a Turing instability.

By simply examining the selection conditions it is possible to make some general statements about the the geometry of TW- and SW-selection parameter regions. However, for the specific type of models (3) we are concerned with, one can immediately see from the form of (50) that all regions in the $\left(\gamma_{2}, \gamma_{3}\right)$ space will have parabolic borders with a tip at the origin where $\gamma_{2}=\gamma_{3}=0$, and that they may only differ in their curvature (second 
derivative). The only two possible qualitatively different configurations of parameter space are shown in Fig. 6. They are separated by the condition $f^{\prime}=g^{\prime}$. For $f^{\prime}>g^{\prime}$, there are regions of parameter space that will support stable travelling waves. However, in this case no stable SWs are possible. We call this configuration a TW-generator. For $f<\dot{g}$, there are also regions of parameter space where standing waves are stable and TWs exist but are unstable. We call this configuration the TW/SW-generator. In a TW-generator SWs exist everywhere where TWs exist. In a TW/SW-generator TWs exist everywhere where SWs exist. In the next section we use this machinery to explore TW vs SW selection in models with a cubic and sigmoidal firing rate nonlinearity.

\subsubsection{Cubic and sigmoidal firing rate function}

The general case of independent $\gamma_{2}$ and $\gamma_{3}$ can be emulated by choosing a cubic nonlinearity $f(u)=\gamma_{1} u+\gamma_{2} u^{2}+\gamma_{3} u^{3}$. Note that in this case there is no truncation of the amplitude equations and it turns out that the amplitude equation predictions match very closely the numerical results even far beyond the bifurcation point. Since the firing rate is now unbounded, patterns outside the existence regions grow unbounded beyond bifurcation from the homogeneous steady state. Choosing a particular function $f$ in effect specifies a locally homeomorphic mapping, $\Gamma$, of the $\left(\gamma_{2}, \gamma_{3}\right)$-plane to a new space of parameters. For the sigmoidal function (2) this space is in fact lower-dimensional. By putting $\bar{\alpha}=\exp (\beta(\bar{u}-h))$ we have

$$
f(\bar{u})=\frac{1}{1+\bar{\alpha}}, f^{\prime}=\frac{\beta \bar{\alpha}}{(1+\bar{\alpha})^{2}}, f^{\prime \prime}(\bar{u})=\frac{\beta^{2} \bar{\alpha}(\bar{\alpha}-1)}{(1+\bar{\alpha})^{3}}, f^{\prime \prime \prime}(\bar{u})=\frac{\beta^{3} \bar{\alpha}\left(\bar{\alpha}^{2}-4 \bar{\alpha}+1\right)}{(1+\bar{\alpha})^{4}} .
$$

At the bifurcation point the transcendental equation $\gamma_{c}=f^{\prime}(\bar{u})$ reduces the number of independent parameters by one. For example we may use this equation to write $h=h(\beta)$ and then express both $\gamma_{2}=f^{\prime \prime}(\bar{u}) / 2$ and $\gamma_{3}=f^{\prime \prime \prime}(\bar{u}) / 6$ as functions of just the gain $\beta$. The relationship between $h$ and $\beta$ (for a fixed $\gamma_{c}=1$ ) is plotted in Fig. 7, right. The parameter portrait on the two branches is identical because the axis of symmetry of $\left(\gamma_{2}, \gamma_{3}\right)$-space maps onto the middle of the graph $h=0$. Note that for $\beta<4 \gamma_{c}$, there is no corresponding value of $h$ which solves the condition to be at the bifurcation point $\gamma_{c}$.

\subsection{Benjamin-Feir instability}

We have determined some aspects of the nonlinear behaviour of the full system from the reduced amplitude equations (47). Basically, the stable solution of the system (47) is 
composed of one (TW) or two (SW) periodic wavetrains moving with speed determined by the linear instability. However, the diffusion term present in the full space-dependent amplitude equations (44) alters somewhat the nature of solutions. The generic instability associated with such diffusion, in an infinite $1 \mathrm{D}$ system, is an instability to long-wave sideband perturbations due to the influence of nearby excited modes on the primary mode. This is the so-called Benjamin-Feir-Eckhaus instability. We will describe it briefly here, for more detail see for example [35].

Let us consider a travelling wave having a wavenumber $k=k_{c}+\epsilon q$, where $q \in(-\sqrt{\delta}, \sqrt{\delta})$, so that $k$ describes the unstable set of modes for $\gamma_{1}=\gamma_{c}+\epsilon^{2} \delta>\gamma_{c}$. The solution to the spatially extended system is $A=r_{1} \mathrm{e}^{i e_{1} \tau+i q \xi}, e_{1}=a_{i}+b_{i} r^{2}-d_{i} q^{2}$. By adding small perturbations to both the magnitude and phase of $A$ we find the condition for stability of the $q$-mode: $b_{r}<0, \quad b_{r} d_{r}+b_{i} d_{i}+2 q^{2} d_{r}^{2}|b|^{2} /\left(r_{1}^{2} b^{2}\right)<0$. One can see that the relation between the self-coupling and the diffusion constants $b$ and $d$ determines the finite width of a set of modes $q$ with centre the primary mode $k_{c}$, which are stable to sideband perturbations. This is defined by the condition

$$
q^{2}<\frac{b_{r} d_{r}+b_{i} d_{i}}{2 d_{r}^{2}|b|^{2}+b_{r} d_{r}\left(b_{r} d_{r}+b_{i} d_{i}\right)} a_{r} b_{r}
$$

A solution with wavenumber outside this band will break up in favour of a wave with wavenumber from inside the stable band. In Fig. 7, left, we show the Fourier transform of a wave solution to the model from Section 3.1 undergoing a Benjamin-Feir-Eckhaus instability. If the condition

$$
b_{r} d_{r}+b_{i} d_{i}>0
$$

holds, there is no stable wavenumber and several different regimes of spatiotemporal chaos can develop depending on parameters as described in [47]. This is commonly referred to as the Benjamin-Feir instability. For the mean-field Ginzburg-Landau equations it is known that if the control parameter is very close to the bifurcation value, the condition (53) also determines the point of destabilisation of the standing wave solution [30]. Note, however, that the validity of this result for SWs is limited by more complicated re-stabilisation boundaries at slightly larger values of $\gamma_{1}$ [46]. We will not consider this here. An example of a Benjamin-Feir instability will be presented later in Section 6 for a model with mexican hat connectivity and spike frequency adaptation (Fig. 11). 


\subsection{Examples revisited}

We apply the theory developed so far to determine the regions of TW-SW selection for the model with axonal delays we looked at in Section 3.1. Again, we will keep $\alpha=1$ and work with $v$ and the nonlinear parameters $\beta$ (neuronal gain) and $h$ (threshold). Using the remarks made in Section 5.1.1 we plot the TW-SW regions in terms of $\beta$ only (see Fig. 7, center). We can see that for all $v$ only travelling waves are stable. Note that for $\beta$ under the dashed line $\beta=4 \gamma_{c}$ we cannot be at the bifurcation point for any $h$ (see Fig. 7, right) and the amplitude equations in that region are not valid. For the range of speeds $v \approx 0.15$ to $v \approx 0.65$ the analysis is also not relevant because another eigenvalue becomes first linearly unstable through a Hopf bifurcation (see Fig. 3). A Benjamin-Feir instability is not observable in this model because the stability boundary coincides almost
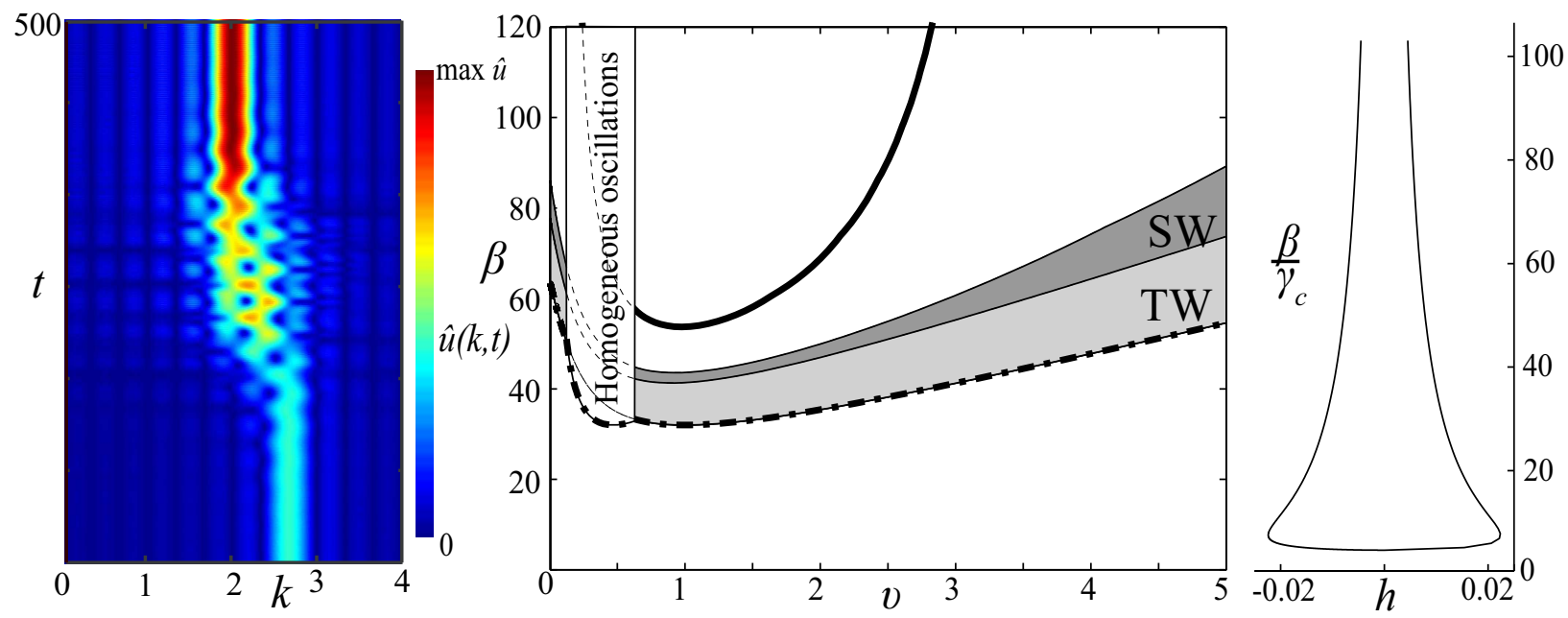

Fig. 7. Left: An example of a Benjamin-Feir-Eckhaus instability in the model of Section 3.1. Parameters are the same as for Fig. 3a with $v=1$ and $\gamma_{1}=\gamma_{c}+2.6$. The primary unstable wavenumber when $\gamma_{1}=\gamma_{c}$ is $k_{c}=2$. Shown is the Fourier transform, $\widehat{u}(k, t)$, of $u(x, t)$ illustrating the active wavenumbers. Initial wave-data with $k=2.64$ can be seen to transition to a pattern with wavenumber $k=1.98$ (the periodic domain size is 19.04). Center: TW-SW selection for the model with axonal delay and inverted wizard hat connectivity. The weakly nonlinear analysis is only valid for $\beta$ above the dashed line and a corresponding $\pm h$ (obtained from the figure on the right). Grey regions denote existence of TWs (light) and SWs (light + dark). The thick line is where $b_{r}=c_{r}$. The domain of Benjamin-Feir stability coincides almost exactly with the TW existence region and so we have not plotted it. Right: The dependence of the gain $\beta$ on the threshold $h$ for the sigmoidal function (2) as determined by the condition to be at the bifurcation point $\gamma_{1}=\gamma_{c}$. 

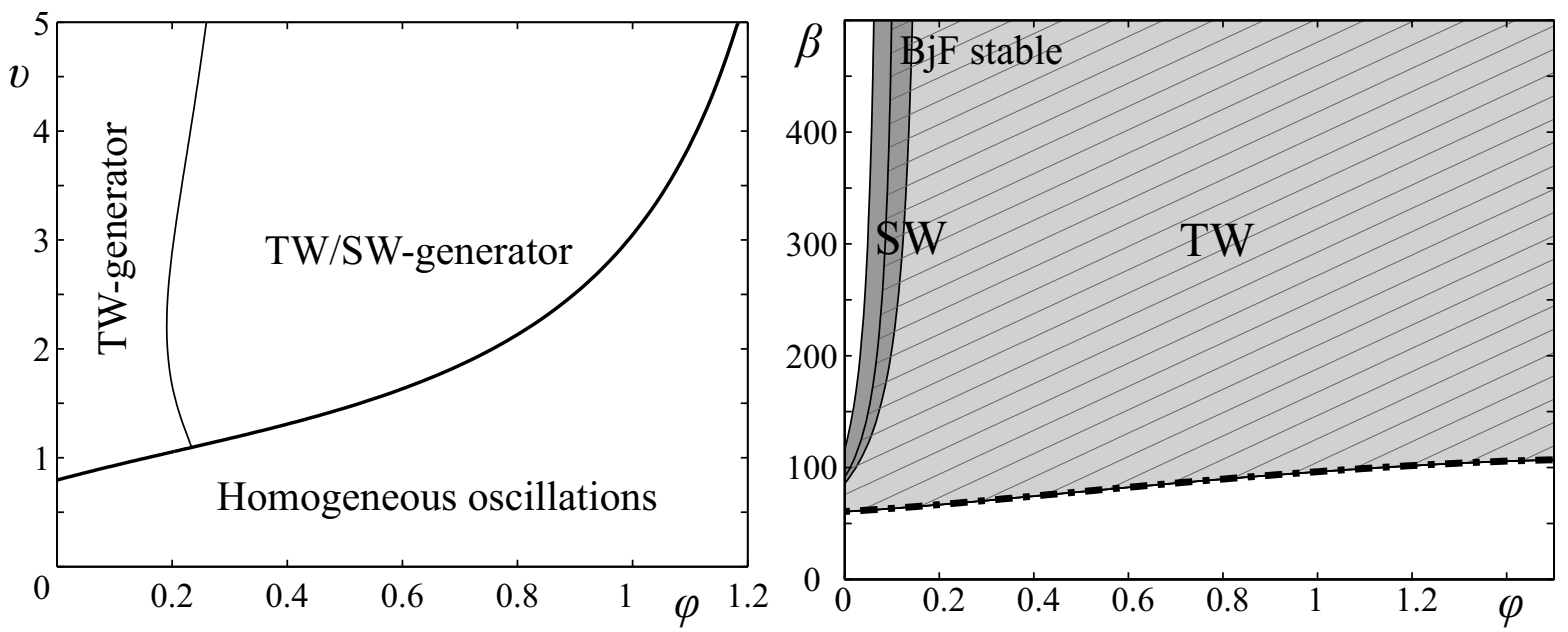

Fig. 8. Left: Two-parameter continuation of the border condition $f^{\prime}=\dot{g}$ that separates TW- and $\mathrm{TW} / \mathrm{SW}$-generators for the model with axo-dendritic delays and $z_{0}=\alpha=\tau=D=1$. For a point $(\phi, v)$ chosen from within the TW-generator (TW/SW-generator) the $\left(\gamma_{2}, \gamma_{3}\right)$ parameter space has the form of Fig. 6 left (right). Right: The regions of existence of TWs (light grey) and SWs (both dark and light grey) and their Benjamin-Feir stability (shaded) for the model with correlated dendritic delays (no axonal delay) and sigmoidal firing rate. TWs are always stable.

exactly with that of travelling waves.

Similarly if we follow the critical curve of the model with dendritic delays with a fixed synaptic site $z_{0}$ (6) (Fig. 5, left) we find that the only stable solutions for every $z_{0}$ are travelling waves. The case of correlated synapses is more interesting and we consider first a cubic nonlinearity. As we vary the synaptic contact parameter $\phi$ we find that there is a value at which behaviour switches between the TW- and TW/SW-generator (i.e. when $\left.f^{\prime}=g\right)$. We can track this point in a second parameter, for example the axonal conduction velocity $v$. We plot this diagram on the left of Fig. 8. Consistent with other models we obtain synchronised homogeneous oscillations for large delay and TWs for small delays. Intermediate between these two extremes we find a TW/SW-generator. However, here the sizes of regions of stable SWs are very small. A sigmoidal nonlinearity stretches the parameter space in a way which makes stable SWs practically unobtainable for this model (see Fig. 8, right). 


\section{Spike frequency adaptation}

In real cortical tissues there is an abundance of metabolic processes whose combined effect is to modulate neuronal response. It is convenient to think of these processes in terms of local feedback mechanisms that modulate synaptic currents. Here we consider a simple model of so-called spike frequency adaptation (SFA), previously discussed in [42]:

$$
u=\eta *\left[K \otimes f \circ u-\gamma_{a} a\right], \quad \partial_{t} a=-a+\int_{-\infty}^{\infty} \mathrm{d} y w_{a}(x-y) f_{a}(u(y, t)),
$$

where $\gamma_{a}>0$ is the strength of coupling to the adaptive field $a$ and $w_{a}(x)=\exp (-|x| / \sigma) / 2 \sigma$ describes the spread of negative feedback. By solving for $a$ with the Green's function $\eta_{a}(t)=\mathrm{e}^{-t} \Theta(t)$ we can reduce the model to a single equation of the form

$$
u=\eta *\left(K \otimes f+K_{a} \otimes f_{a}\right) \circ u
$$

where $K_{a}(x, t)=-\eta_{a}(t) w_{a}(x)$ and the coefficient $\gamma_{a}$ has been incorporated into the nonlinearity $f_{a}$. Using this notation it is apparent that both our linear and weakly nonlinear analysis can be readily extended. The dispersion relation for a model with SFA can be written in the form $E_{1}(k, \lambda)=1$ with $E_{i}(k, \lambda)=\widetilde{\eta}(\lambda)\left(\gamma_{i} \widehat{K}(k, \lambda)+\gamma_{a_{i}} \widehat{K}_{a}(k, \lambda)\right)$ where $\gamma_{a_{n}}=f_{a}^{(n)}(\bar{u})$. Then, the coefficients of the mean-field Ginzburg-Landau equation are calculated as

$$
\begin{aligned}
a & =-\delta D \\
b & =-D\left(3 E_{3}^{11}+2 E_{2}^{11}\left(2 C_{00}+C_{22}\right)\right) \\
c & =-2 D\left(3 E_{3}^{11}+2 E_{2}^{11}\left(C_{00}+C_{02}+C_{20}\right)\right), \\
d & =-\frac{1}{2} D\left(\frac{\partial}{\partial i k}-v_{g} \frac{\partial}{\partial i \omega}\right)^{2} E_{c}^{11} \\
D & =\left(\frac{\partial E_{c}^{11}}{\partial i \omega}\right)^{-1}, \quad C_{m n}=\frac{E_{2}^{m n}}{1-E_{c}^{m n}}, \quad v_{g}=\frac{\partial \omega}{\partial k}
\end{aligned}
$$

with $E_{i}^{m n}=E_{i}\left(n k_{c}, i m \omega_{c}\right)$, and all quantities above evaluated at the critical point where $(k, \omega)=\left(k_{c}, \omega_{c}\right)$ and $E_{1}=E_{c}$.

Consider the model with axonal delay given by $K(x, t)=(1-|x|) \mathrm{e}^{-|x|} \delta(t-|x| / v)$ in the context of linear adaptation $\left(f_{a}(u)=\gamma_{a} u\right)$. The linear instability portrait with respect to adaptation strength $\gamma_{a}$ is plotted in Fig. 9, left for $v=1$ and $\sigma=0$ (representing local feedback). The case with $\sigma \neq 0$ (non-local feedback) is qualitatively similar. With increasing $\gamma_{a}$ the main effect, on all critical curves (such as those seen in Fig. 3), is to move 

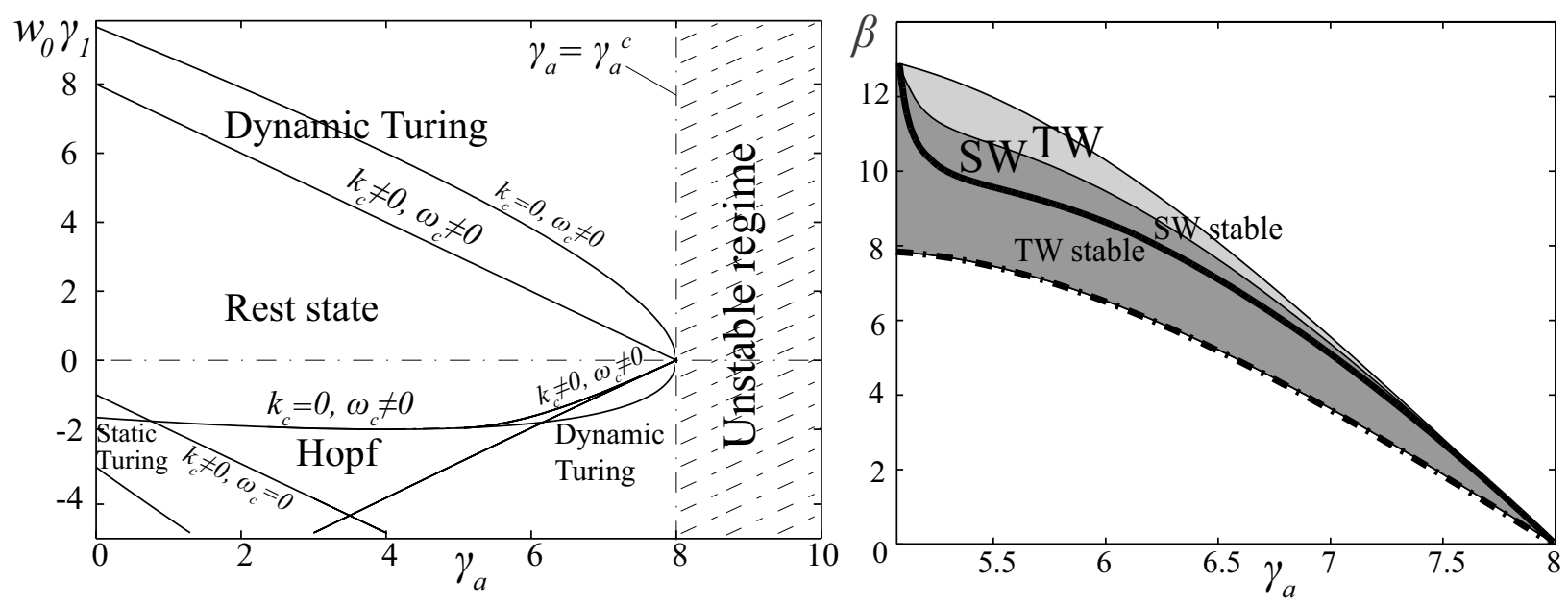

Fig. 9. Left: The linear instabilities of the model with axonal delay $v=1$ and localised $(\sigma=0)$ linear adaptation with strength $\gamma_{a}$. Right: TW-SW selection along the Turing-Hopf instability curve for standard wizard hat on the left. Here we have a TW/SW-generator and for visibility shades have been chosen differently from previous plots. SWs exist in the dark grey area only. TWs exist in both the light and dark grey areas. The thick line separates stable TWs from stable SWs. Both TWs and SWs are Benjamin-Feir stable.

them toward $\gamma_{1}=0$. For $\gamma_{a}$ larger than some critical value $\gamma_{a}^{c}$ the homogeneous steady state becomes unstable. In this case the majority of wavemodes are excited and solutions grow to infinity. For $w_{0}<0$ (and the physically sensible choice $\gamma_{1}>0$ ) we see that a dynamic instability can occur for sufficiently large $\gamma_{a}<\gamma_{a}^{c}$. This statement is true for all $v$. Hence, dynamic instabilities are possible in a model with short range excitation and long range inhibition provided there is sufficiently strong adaptive feedback. Indeed, this observation has previously been made by Hansel and Sompolinsky [48]. In fact, Curtu and Ermentrout [29] discovered homogenous oscillations, travelling waves and standing waves in the neighbourhood of a Takens-Bogdanov (TB) bifurcation in a model with Mexican hat connectivity and feedback lacking space-dependent delays $\left(v^{-1}=0\right)$. The dependence of this TB point on $v$ and $\gamma_{a}$ in a model with standard wizard hat connectivity is shown in Fig. 10. It first appears for $v \approx 6.27$ at $\gamma_{a} \approx 2.51$. In contrast to previous models we have studied, the regions of stable SWs are large for the general cubic case and exist even for the sigmoidal firing rate (see Fig. 9 right). Another new feature in the presence of SFA is the appearance of parameter regimes at large $v$ for which TWs and SWs are BenjaminFeir unstable. Solutions of all models without SFA that we studied were verified to be Benjamin-Feir stable. An example of such instability of SW in a model with SFA is shown 

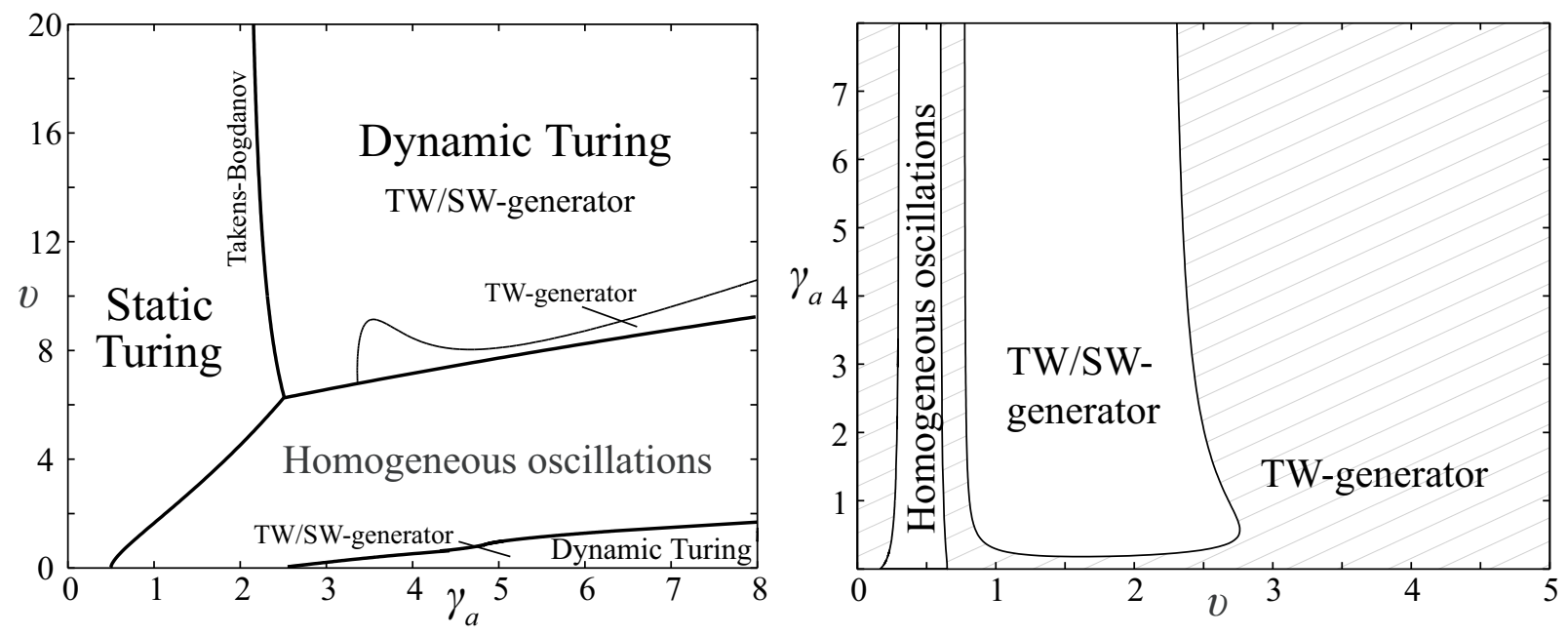

Fig. 10. Left: Organisation of emergent dynamics in a model with axonal delays and spike-frequency adaptation with standard wizard hat connectivity $\left(w_{0}<0\right)$. Here $\sigma=0$, $\alpha=\alpha_{a}=1$. Right: The dynamics of the model with axonal delay and nonlinear adaptation with strength $\gamma_{a}$ and $\sigma=0$.

in Fig. 11. However, we were not able to observe chaotic solutions as one might expect from the properties of the system's normal form. The questionas to why the transition settles to modulated ordered solutions remains open.

Let us briefly consider an example of nonlinear adaptation with the choice $f_{a}=\gamma_{a} f$ in (55). In the case of purely local feedback $(\sigma=0)$ the linear instability portrait for the model of Section 3.1 does not change qualitatively when $\gamma_{a}$ is increased from zero, although all instability curves are moved towards zero for both $w_{0}>0$ and $w_{0}<0$. Unlike the linear adaptation model they do not hit $\gamma_{1}=0$ but instead tend assymptotically to it. Thus there is no "unstable regime" where all modes are excited. A region of TW/SWgenerator appears for medium speeds, shown in Fig. 10, though the SW bands in $\left(\gamma_{2}, \gamma_{3}\right)$ space are very small. However, they significantly widen for nonlocal feedback and can also be observed for the case of sigmoidal firing rates.

\section{Discussion}

In this paper we have studied pattern formation in a broad class of neural field models. These models provide minimal descriptions of neural tissue with biological features that include synaptic and dendritic processing, the effects of axonal delays, and spike frequency adaptation. Importantly we have written them using the language of integral equations 

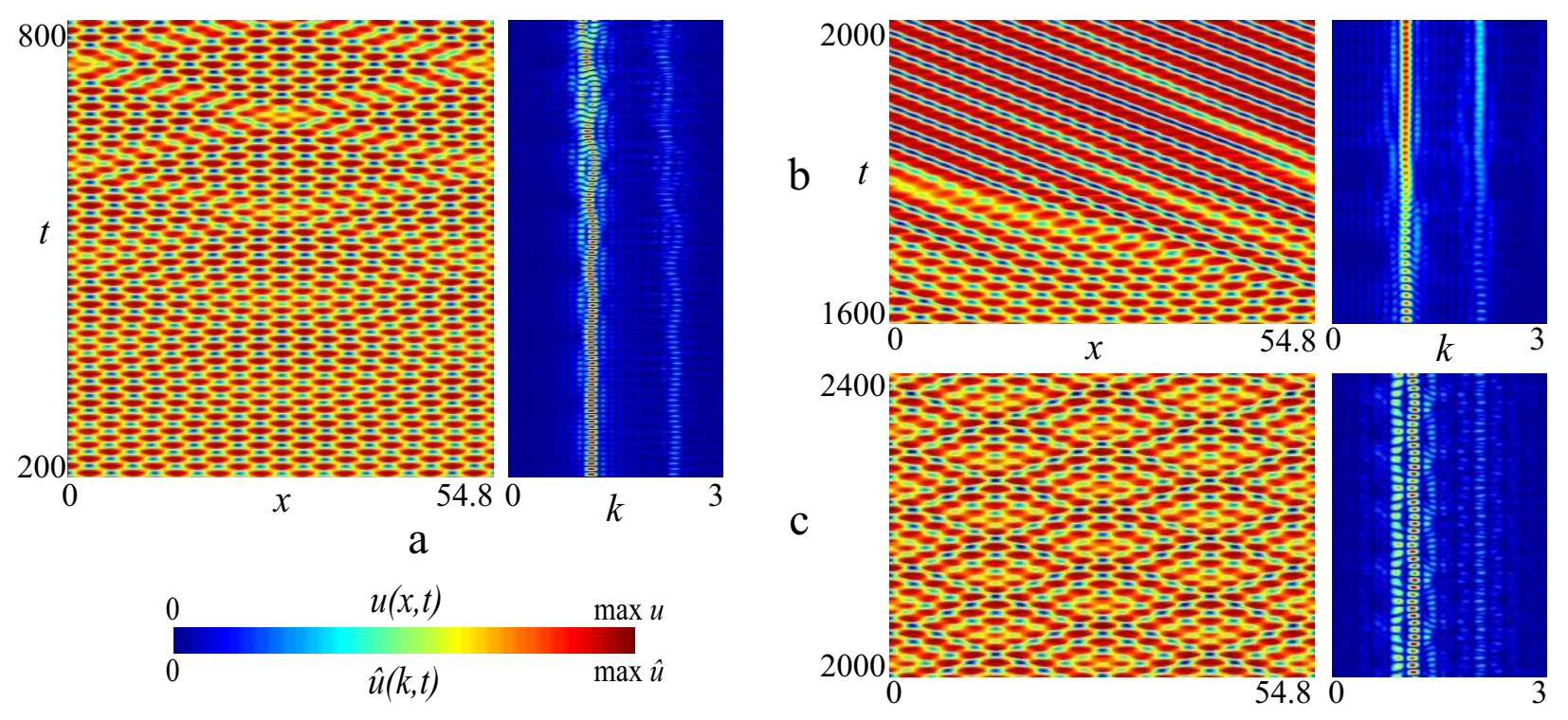

Fig. 11. A Benjamin-Feir instability of standing waves in the model with linear SFA with a cubic nonlinearity. Parameters: $v=8, \gamma_{a}=2.66, \gamma_{1}=\gamma_{c}+0.1, \gamma_{2}=-1.3, \gamma_{3}=-0.4$. The initial condition favours a standing wave with the primary unstable wavenumber $k_{c}=1.144$, but wavenumbers in its neighbourhood start to grow (a). After some time new nonlinear stability conditions come into play which, depending on the initial condition (respectively $k=1.0395$ and $k=k_{c}$ ), might let only a single wavenumber survive (b) or stabilise a more complicated configuration (c). On the Fourier space plots one can also see the appearance of a spatial resonance. Here, wavenumbers which are multiples of the dominant ones also become partially excited.

and spatio-temporal kernels. By pursuing a systematic multiple scales approach for deriving amplitude equations in an integral framework we have been able to recover a number of known results about pattern formation in neural fields, and more importantly been able to treat the biologically important case of space-dependent delays for the first time. These delays arise naturally in models with axons and dendrites and are treated from a mathematical perspective by choosing an appropriate spatio-temporal kernel $K(x, t)$ for the integral model. In fact because our approach does not depend upon detailed properties of $K(x, t)$ the techniques we have developed can also cope with time varying synaptic connections, such as would be the case if a network were undergoing some form of learning. The derivation of the appropriate amplitude equations for group velocity of $O(1)$ has allowed us to treat the selection problem for standing vs travelling waves in models with both axonal [18] and dendritic delays [17], and to also recover and extend the original work of Curtu and Ermentrout [29] on linear spike frequency adaptation. Although the latter authors have used similar methods to ours to find instances of standing waves, one point 
worth making for all the models studied in this paper is that the windows of parameter space that support stable standing waves are, in fact, very small. Our analysis has enabled us to first delimit such regions and second to consider how they may be maximized. For example in the case of nonlinear spike frequency adaptation we find that increasing the spatial extent of modulating currents is one such mechanism for promoting the robustness of stable standing waves under parameter variation.

A number of extensions of the work in this paper readily suggest themselves, including

i) the study of two-population models [1], ii) the effects of heterogeneity [49,28], iii) the treatment of dynamic thresholds [50], iv) the effects of distributed axonal speeds [51] and v) the extension to two spatial dimensions. These are topics of ongoing study and will be reported on elsewhere.

\section{Acknowledgements}

SC would like to acknowledge ongoing support from the EPSRC through the award of an Advanced Research Fellowship, Grant No. GR/R76219.

\section{References}

[1] H R Wilson and J D Cowan. Excitatory and inhibitory interactions in localized populations of model neurons. Biophysical Journal, 12:1-24, 1972.

[2] D Golomb and Y Amitai. Propagating neuronal discharges in neocortical slices: Computational and experimental study. Journal of Neurophysiology, 78:1199-1211, 1997.

[3] J Y Wu, L Guan, and Y Tsau. Propagating activation during oscillations and evoked responses in neocortical slices. Journal of Neuroscience, 19:5005-5015, 1999.

[4] R Miles, R D Traub, and R K S Wong. Spread of synchronous firing in longitudinal slices from the CA3 region of Hippocampus. Journal of Neurophysiology, 60:1481-1496, 1995.

[5] X Huang, W C Troy, Q Yang, H Ma, C R Laing, S J Schiff, and J Wu. Spiral waves in disinhibited mammalian neocortex. The Journal of Neuroscience, 24:9897-9902, 2004.

[6] B W Connors and Y Amitai. Generation of epileptiform discharges by local circuits in neocortex. In P A Schwartzkroin, editor, Epilepsy: Models, Mechanisms and Concepts, pages 388-424. Cambridge University Press, 1993. 
[7] G B Ermentrout and J D Cowan. A mathematical theory of visual hallucination patterns. Biological Cybernetics, 34:137-150, 1979.

[8] P Tass. Cortical pattern formation during visual hallucinations. Journal of Biological Physics, 21:177-210, 1995.

[9] P C Bressloff and J D Cowan. Spontaneous pattern formation in primary visual cortex. In A. Champneys S. J. Hogan and B. Krauskopf, editors, Nonlinear dynamics: where do we go from here? Institute of Physics, Bristol, 2002.

[10] S Amari. Dynamics of pattern formation in lateral-inhibition type neural fields. Biological Cybernetics, 27:77-87, 1977.

[11] K Kishimoto and S Amari. Existence and stability of local excitations in homogeneous neural fields. Journal of Mathematical Biology, 7:303-318, 1979.

[12] G B Ermentrout and J B McLeod. Existence and uniqueness of travelling waves for a neural network. Proceedings of the Royal Society of Edinburgh, 123A:461-478, 1993.

[13] D J Pinto and G B Ermentrout. Spatially structured activity in synaptically coupled neuronal networks: I. Travelling fronts and pulses. SIAM Journal on Applied Mathematics, 62:206-225, 2001.

[14] S Coombes. Waves, bumps and patterns in neural field theories. Biological Cybernetics, 93:91-108, 2005.

[15] P L Nunez. The brain wave equation: a model for the EEG. Mathematical Biosciences, 21:279-297, 1974.

[16] V K Jirsa and H Haken. A derivation of a macroscopic field theory of the brain from the quasi-microscopic neural dynamics. Physica D, 99:503-526, 1997.

[17] P C Bressloff. New mechanism for neural pattern formation. Physical Review Letters, 76:4644-4647, 1996.

[18] A Hutt, M Bestehorn, and T Wennekers. Pattern formation in intracortical neuronal fields. Network, 14:351-368, 2003.

[19] B A McGuire, C D Gilbert, P K Rivlin, and T N Wiesel. Targets of horizontal connections in macaque primary visual cortex. Journal of Comparative Neurology, 305:370-392, 1991.

[20] P-A Salin and J Bullier. Corticocortical connections in the visual system: structure and function. Physiological Reviews, 75:107-154, 1995. 
[21] C D Gilbert, A Das, M Ito, M Kapadia, and G Westheimer. Spatial integration and cortical dynamics. Journal of Comparative Neurology, 93:615-622, 1996.

[22] A Hutt. Local excitation-lateral inhibition interaction yields wave instabilities in spatial systems involving finite propagation delay. arXiv:nlin/0610037, 2006.

[23] A Roxin, N Brunel, and D Hansel. Role of delays in shaping spatiotemporal dynamics of neuronal activity in large networks. Physical Review Letters, 28:357-376, 2005.

[24] P C Fife. Mathematical Aspects of Reacting and Diffusing Systems. Lecture Notes in Biomathematics. Springer, Berlin, 1979.

[25] F H Busse. Nonlinear properties of thermal convection. Rep. Prog. Phys., 41:1929-1967, 1978.

[26] B G Ermentrout. Stable small-amplitude solutions in reaction-diffusion systems. Quart. Appl. Math., 39:61-86, 1981.

[27] B G Ermentrout and J Cowan. Secondary bifurcation in neuronal nets. SIAM Journal on Applied Mathematics, 39:323-340, 1980.

[28] P C Bressloff. Spatially periodic modulation of cortical patterns by long-range horizontal connections. Physica D, 185:131-157, 2003.

[29] R Curtu and B Ermentrout. Pattern formation in a network of excitatory and inhibitory cells with adaptation. SIAM Journal on Applied Dynamical Systems, 3:191-231, 2004.

[30] E Knobloch and J de Luca. Amplitude equations for travelling wave convection. Journal of Nonlinearity, 3:975-980, 1990.

[31] H R Wilson and J D Cowan. A mathematical theory of the functional dynamics of cortical and thalamic nervous tissue. Kybernetik, 13:55-80, 1973.

[32] V K Jirsa and H Haken. Field theory of electromagnetic brain activity. Physical Review Letters, 77:960-963, 1996.

[33] P C Bressloff and S Coombes. Physics of the extended neuron. International Journal of Modern Physics B, 11:2343-2392, 1997.

[34] G. M. Shepherd, editor. The Synaptic Organization of the Brain. Oxford University Press, 1990.

[35] R Hoyle. Pattern formation: An introduction to methods. Cambridge University Press, 2006. 
[36] A C Newell. The dynamics and analysis of patterns. In H F Nijhout, L Nadel, and D L Stein, editors, Pattern formation in the physical and biological sciences, pages 201-268. Addison-Wesley, Santa Fe Institute, 1997.

[37] M C Cross and P C Hohenberg. Pattern formation outside of equilibrium. Reviews of Modern Physics, 65(3):851-1112, 1935.

[38] D Walgraef. Spatio-temporal pattern formation. Springer-Verlag, 1997.

[39] G B Ermentrout. Asymptotic behavior of stationary homogeneous neuronal nets. In S Amari and M A Arbib, editors, Competition and cooperation in neural nets, pages 57-70. Springer, 1982.

[40] P C Bressloff. Les Houches Lectures in Neurophysics, chapter Pattern formation in visual cortex, pages 477-574. Elsevier.

[41] N A Venkov. Dynamics of neural field models. PhD thesis, School of Mathematical Sciences, University of Nottingham. In preparation.

[42] S Coombes, G J Lord, and M R Owen. Waves and bumps in neuronal networks with axo-dendritic synaptic interactions. Physica D, 178:219-241, 2003.

[43] A Hutt and F M Atay. Analysis of nonlocal neural fields for both general and gammadistributed connectivities. Physica D, 203:30-54, 2005.

[44] G Helmberg. Introduction to Spectral theory in Hilbert space. North-Holland Publishing Company, 1969.

[45] R D Pierce and C E Wayne. On the validity of mean-field amplitude equations for counterpropagating wavetrains. Journal of Nonlinearity, 8:769-779, 1995.

[46] H Riecke and L Kramer. The stability of standing waves with small group velocity. Physica D, 137:124-142, 2000 .

[47] B I Shraiman, A Pumir, W van Saarloos, P C Hohenberg, H Chaté, and M. Holen. Spatiotemporal chaos in the one-dimensional complex Ginzburg-Landau equation. Physica $D, 57(3-4): 241-248,1992$.

[48] D Hansel and H Sompolinsky. Methods in Neuronal Modeling, From Ions to Networks (2nd Edition), chapter Modeling Feature Selectivity in Local Cortical Circuits, pages 499-567. MIT Press, 1998.

[49] V K Jirsa and J A S Kelso. Spatiotemporal pattern formation in neural systems with heterogeneous connection topologies. Physical Review E, 62:8462-8465, 2000. 
[50] S Coombes and M R Owen. Bumps, breathers, and waves in a neural network with spike frequency adaptation. Physical Review Letters, 94:148102, 2005.

[51] A Hutt and F M Atay. Effects of distributed transmission speeds on propagating activity in neural populations. Physical Review E, 73:021906, 2006. 